\title{
Orthotropic active strain models for the numerical simulation of cardiac biomechanics
}

\author{
Simone Rossi ${ }^{1,2}$, Ricardo Ruiz-Baier ${ }^{1, *, \dagger}$, Luca F. Pavarino ${ }^{3}$ and Alfio Quarteroni ${ }^{1,4}$ \\ ${ }^{1}$ CMCS-MATHICSE-SB, Ecole Polytechnique Fédérale de Lausanne, CH-1015 Lausanne, Switzerland \\ ${ }^{2}$ Departamento de Matemática, Instituto Superior Técnico, Av. Rovisco Pais 1, 1049-001 Lisbon, Portugal \\ ${ }^{3}$ Dipartimento di Matematica, Universitá di Milano, Via Saldini 50, 20133 Milano, Italy \\ ${ }^{4}$ MOX - Modellistica e Calcolo Scientifico Dipartimento di Matematica "F. Brioschi”, Politecnico di Milano, \\ via Bonardi 9, 20133 Milano, Italy
}

\begin{abstract}
SUMMARY
A model for the active deformation of cardiac tissue considering orthotropic constitutive laws is introduced and studied. In particular, the passive mechanical properties of the myocardium are described by the Holzapfel-Ogden relation, whereas the activation model is based on the concept of active strain. There, an incompatible intermediate configuration is considered, which entails a multiplicative decomposition between active and passive deformation gradients. The underlying Euler-Lagrange equations for minimizing the total energy are written in terms of these deformation factors, where the active part is assumed to depend, at the cell level, on the electrodynamics and on the specific orientation of the cardiomyocytes. The active strain formulation is compared with the classical active stress model from both numerical and modeling perspectives. The well-posedness of the linear system derived from a generic Newton iteration of the original problem is analyzed, and different mechanical activation functions are considered. Taylor-Hood and MINI finite elements are used in the discretization of the overall mechanical problem. The results of several numerical experiments show that the proposed formulation is mathematically consistent and is able to represent the main features of the phenomenon, while allowing savings in computational costs. Copyright (C) 2012 John Wiley \& Sons, Ltd.
\end{abstract}

Received 30 May 2011; Accepted 17 January 2012

KEY WORDS: cardiac mechanics; active strain formulation; finite element discretization; nonlinear incompressible elasticity

\section{INTRODUCTION}

Mathematical modeling of living soft tissue presents several challenges originating from anisotropic material behavior, nonlinear equations, geometrical complexity, heterogeneity, difficult in vivo measurements, and the presence of phenomena taking place at different scales, among many others [1]. From the viewpoint of solid mechanics analysis, these materials can be regarded as anisotropic and viscoelastic, and they can experience non-linear elastic large deformations both under normal physiological conditions and injury [2].

In this framework, a subject of crucial interest is the modeling of the mechanical properties of cardiac tissue. The ventricular wall can suffer changes in thickness of up to $40 \%$ during contraction [3]; and thus, the governing equations of continuum mechanics need to be cast in the framework

\footnotetext{
*Correspondence to: Ricardo Ruiz-Baier, CMCS-MATHICSE-SB, Ecole Polytechnique Fédérale de Lausanne, CH-1015

Lausanne, Switzerland.

†E-mail: ricardo.ruiz@epfl.ch

Copyright @ 2012 John Wiley \& Sons, Ltd.
} 
of nonlinear elasticity (no assumption of infinitesimal strain) and to use an anisotropic description based on the direction of the fibers and collagen sheets compound that form the tissue $[4,5]$. In contrast to other hyperelastic materials such as rubber, a key intrinsic feature of living tissues is that they not only passively balance external forces but they actively deform without the need of external loads. Experimental studies [6,7] have demonstrated that several ionic channels in the myocardium are activated by cell stretch and volume changes. Conversely, the fibers of the heart muscle are able to contract in response to active mechanisms mainly driven by the release of intracellular calcium at the microscopic level [8].

This phenomenon is usually modeled either by incorporating the activation as an additive contractile force in the stress tensor in the current or reference configuration [4,9-11] (hereafter, this strategy will be referred to as the active stress model), or more recently, by assuming that an intermediate elastic configuration exists between the initial and current states, which translates in a multiplicative decomposition of the deformation gradient into active and passive factors [12-14] (we will refer to this as the active strain model). Both models need a precise description of an activation function driving the kinematics of the mechanical contraction. The active strain formulation has been previously applied to isotropic and transversely isotropic constitutive laws $[12,13]$, but a deeper explanation of the method from both mathematical and modeling viewpoints is still missing. Transversely isotropic and fully orthotropic models for active contraction can be found in the literature $[5,15]$ but addressing active stress formulations mainly. Here, we will explore some consequences of adopting an active strain framework, where a key advantage is that the properties of frame invariance and rank-one ellipticity are naturally inherited from those of the passive constitutive law [16].

Numerical solutions for incompressible and nearly incompressible linear elasticity problems are usually based on finite element methods. The fulfillment of the inf-sup condition [17] is required at both continuous and discrete levels, ensuring the unique solvability and stability of the problem. When it comes to nonlinear elasticity, a standard approach consists in applying a linearization via the Newton method; and at each Newton iteration, the linearized problem should verify the corresponding stability condition $[18,19]$. In the context of activated elastic materials, we found that these conditions are satisfied for physiological values assumed by the activation function.

The main objectives of this paper deal with the establishment, implementation, and testing of an orthotropic model for cardiac contraction on the basis of an active strain decomposition. Our model addresses the coupling of an active transversely isotropic mechanical description at the cell level, with an orthotropic constitutive law for incompressible tissue at the macroscopic level. The fundamental idea used in this approach consists in the definition of a new strain energy function that is able to describe the active deformations dictated by the definition of an active deformation gradient. Moreover, the main differences with the active stress model are addressed in detail showing how the active strain naturally modifies passive constitutive laws. In addition, finite element discretizations using Taylor-Hood and MINI elements are proposed and illustrated with several numerical examples that allow not only an assessment of the performance of the methods but also shed some light on the applicability of the model in comparison with experimental observations.

We have arranged the contents of this paper as follows. In Section 2, we summarize some notions for general elasticity problems, and we provide a precise weak formulation. We describe the main building blocks for the cardiac finite elasticity, including both active stress and active strain approaches. In particular, we introduce the active strain formulation in the framework of orthotropic constitutive laws. Section 3 presents a theoretical comparison between active strain and active stress analyzing the final form of the stress tensor for some possible material laws, and we also discuss the fulfillment of strong ellipticity. In Section 4, we show that the linearization of the considered system leads naturally to a saddle point problem, for which we provide a well-posedness analysis. Section 5 describes the finite element methods used and the incremental load algorithm essential to ensure convergence of the Newton scheme. In Section 6, we collect numerical experiments in two and three dimensions, which confirm the discussion in Section 3, and allow us to compare the two active strain and stress formulations. Some conclusions and extensions are discussed in Section 7. 


\section{MATHEMATICAL MODEL FOR CARDIAC MECHANICS}

\subsection{Hyperelastic behavior of passive myocardium}

In the sequel, $\boldsymbol{x}$ will denote the current position of a material particle of a bounded body $\mathcal{B}$ that was originally placed at $\mathbf{X}$ in the reference configuration $\mathcal{B}_{0} \subset \mathbb{R}^{d}, d=2,3$. The motion of the elastic body is defined using the smooth one-to-one map $\varphi: \mathcal{B}_{0} \rightarrow \mathcal{B} \subset \mathbb{R}^{d}, \boldsymbol{\varphi}(\mathbf{X})=\mathbf{X}+\boldsymbol{u}(\mathbf{X})$, where $\boldsymbol{u}$ denotes the displacement vector $\boldsymbol{u}=\boldsymbol{x}-\mathbf{X}$. Strain measures can be obtained from the deformation gradient tensor $\mathbf{F}=\mathbf{I}+\nabla \boldsymbol{u}$ : the right and left Cauchy-Green deformation tensors $\mathbf{C}=\mathbf{F}^{T} \mathbf{F}$, $\mathbf{B}=\mathbf{F F}^{T}$, respectively, where $\mathbf{I}$ is the second-order identity tensor and $\nabla$ stands for the gradient with respect to material coordinates. By $J=\operatorname{det} \mathbf{F}$, we denote the volume map. The constitutive law for an isotropic material can be written using the isotropic invariants

$$
I_{1}:=\operatorname{tr} \mathbf{C}, I_{2}:=\frac{1}{2}\left(I_{1}^{2}-\operatorname{tr}^{2}\right), I_{3}:=\operatorname{det} \mathbf{C}=J^{2} .
$$

Following [20], for any fixed directions $\mathbf{i}$ and $\mathbf{j}$, we define the direction-dependent invariants

$$
I_{4, i}=\mathbf{i} \cdot(\mathbf{C} \mathbf{i}), I_{5, i}=\mathbf{i} \cdot\left(\mathbf{C}^{2} \mathbf{i}\right), I_{8, i j}=\mathbf{i} \cdot(\mathbf{C} \mathbf{j}) .
$$

In a macroscopic description of the cardiac tissue, we assume that the material is hyperelastic; and hence, the measures of stress are obtained by differentiating a pseudo-strain energy $\mathcal{W}$ with respect to strain.

The myocardium is a compressible medium, as observed by Kamgoue et al. [21], because it is perfused with blood. During a contraction cycle, the small vessels supplying oxygen to the cells are squeezed, and the blood is let out so that the total volume (including the blood chambers) is not preserved. This phenomenon can be modeled by considering the tissue as a mixture of incompressible solid and incompressible fluid [22]. However, in the present study, we focus on the hyperelastic incompressible behavior of the heart muscle, neglecting viscoelastic and poroelastic effects. For the incompressibility of the material to be incorporated, an isochoric strain energy can be defined as

$$
\mathcal{W}_{\text {inc }}=\mathcal{W}(\boldsymbol{u})-p(J-1),
$$

where $\mathcal{W}(\boldsymbol{u})$ describes the material properties and $p=p(\mathbf{X})$ is the Lagrange multiplier arising from the imposition of the constraint $J=1$. We will use a constitutive law proposed by Holzapfel and Ogden [20] for myocardial tissue. It takes into account material orthotropy (see also [2,5]) that is due to the presence of preferred directions of alignment for cardiac cells and layers of collagen in the tissue. The invariant-based nature of this law allows invariance with respect to the frame of reference, and it generalizes other constitutive models. Neo-Hookean, exponential and polynomial laws [1] can be retrieved by modifying the material parameters at the level of the stress tensor. The energy function is given by

$$
\mathcal{W}(\boldsymbol{u})=\frac{a}{2 b} \exp \left(b\left[I_{1}-d\right]\right)+\sum_{i=f, s} \frac{a_{i}}{2 b_{i}}\left[\exp \left(b_{i}\left[I_{4, i}-1\right]^{2}\right)-1\right]+\frac{a_{f_{s}}}{2 b_{f_{s}}} \exp \left(b_{f_{s}} I_{8, f_{S}}^{2}-1\right),
$$

where the sub-indices $f$ and $s$ refer to the fibers and collagen sheets axes, $\mathbf{f}_{0}$ and $\mathbf{s}_{0}$, respectively (Figure 1).

The quantities $a, a_{f}, a_{s}, a_{f s}, b, b_{f}, b_{s}, b_{f_{s}}$ are experimentally fitted material parameters (Table I), and the invariants in (2.1) can be expressed as

$$
I_{4, f}:=\mathbf{f} \cdot \mathbf{f}, I_{4, s}:=\mathbf{s} \cdot \mathbf{s}, I_{8, f_{s}}:=\mathbf{f} \cdot \mathbf{s},
$$

where $\mathbf{f}:=\mathbf{F f}_{0}$ and $\mathbf{s}:=\mathbf{F s}_{0}$ are vectors representing the directions of fibers and sheets in the deformed configuration. The Cauchy stress tensor $\mathbf{T}=J^{-1}(\partial \mathcal{W} / \partial \mathbf{F}) \mathbf{F}^{T}$ assumes the form

$$
\begin{aligned}
\mathbf{T}=a & \exp \left(b\left[I_{1}-d\right]\right) \mathbf{B}-p \mathbf{I}+2 a_{f}\left(I_{4, f}-1\right) \exp \left(b_{f}\left[I_{4, f}-1\right]^{2}\right) \mathbf{f} \otimes \mathbf{f} \\
& +2 a_{s}\left(I_{4, s}-1\right) \exp \left(b_{s}\left[I_{4, s}-1\right]^{2}\right) \mathbf{s} \otimes \mathbf{s}+2 a_{f_{s}} I_{8, f_{s}} \exp \left(b_{f_{s}} I_{8, f_{s}}^{2}\right)[\mathbf{f} \otimes \mathbf{s}+\mathbf{s} \otimes \mathbf{f}] .
\end{aligned}
$$




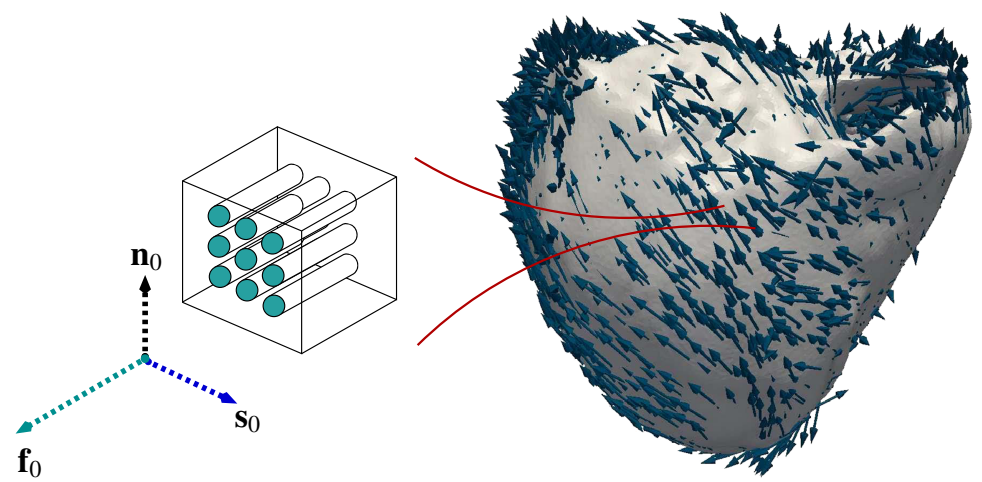

Figure 1. Sketch of portion of the cardiac tissue. Here, $\mathbf{f}_{0}, \mathbf{s}_{0}$ and $\mathbf{n}_{0}$ denote the fibers, sheets, and sheet-normal directions, respectively.

Table I. Parameter values for the Holzapfel-Ogden material law [20, 23].

\begin{tabular}{cccccccc}
\hline$a$ & $a_{f}$ & $a_{s}$ & $a_{f s}$ & $b$ & $b_{f}$ & $b_{s}$ & $b_{f s}$ \\
\hline $0.496[\mathrm{KPa}]$ & $15.193[\mathrm{KPa}]$ & $3.283[\mathrm{KPa}]$ & $0.662[\mathrm{KPa}]$ & 20.417 & 11.176 & 7.209 & 9.466 \\
\hline
\end{tabular}

As a measure of stress in the reference configuration, we use the first Piola-Kirchhoff tensor $\mathbf{P}=\partial \mathcal{W} / \partial \mathbf{F}=J \mathbf{T F}^{-T}$, representing the force per unit of undeformed area acting on the deformed body $\mathcal{B}$. When $\mathcal{B}$ is under a given load $\ell$ per unit volume in the reference configuration, according to the principle of stationary potential energy, the total energy satisfies

$$
\int_{\mathcal{B}_{0}} \mathcal{W}(\boldsymbol{u}, p)=\int_{\mathcal{B}_{0}} \boldsymbol{\ell} \cdot \boldsymbol{u} .
$$

Therefore, the Euler-Lagrange equations written in a mixed form read: Find $\boldsymbol{u} \in V, p \in Q$ such that

$$
\begin{gathered}
\int_{\mathcal{B}_{0}} \mathbf{P}(\boldsymbol{u}, p): \nabla \boldsymbol{v}=\int_{\mathcal{B}_{0}} \boldsymbol{\ell} \cdot \boldsymbol{v} \quad \forall \boldsymbol{v} \in V, \\
\int_{\mathcal{B}_{0}}(J-1) q=0 \quad \forall q \in Q .
\end{gathered}
$$

System (2.4) is completed with homogeneous Dirichlet boundary data on $\Gamma_{D} \subset \partial \mathcal{B}_{0}$ for displacements and stress free boundary conditions on $\Gamma_{N}=\partial \mathcal{B} \backslash \Gamma_{D}$. Here, $V=\left[H_{D}^{1}\left(\mathcal{B}_{0}\right)\right]^{d}=\{\boldsymbol{v} \in$ $\left.H^{1}\left(\mathcal{B}_{0}\right)^{d}:\left.\boldsymbol{v}\right|_{\Gamma_{D}}=\mathbf{0}\right\}$ and $Q=L^{2}\left(\mathcal{B}_{0}\right)$ are admissible spaces for displacement and pressure fields, respectively. Notice that the incompressibility constraint has not yet been enforced in the momentum equation.

The solvability of (2.4) is ensured under certain conditions on the total energy (such as differentiability in the Gâteaux sense) as presented in detail in the early paper of Le Tallec [24, Th. 3.1].

\subsection{Active response based on a stress decomposition}

A common approach to include the dynamics of the active forces within the tissue consists in adding their contribution directly to the stress tensor. Let

$$
\mathbf{P}_{A}=T_{\mathrm{A}} \mathbf{f} \otimes \mathbf{f}_{0}
$$

represent an active stress, microscopically due to biochemical reactions inside the sarcomeres, of magnitude $T_{\mathrm{A}}$ in the direction of the fibers in the reference configuration. Multi-axial active stresses have been also proposed in $[5,15]$, to capture observed experimental results. Nonetheless, we will 
not define here active forces in the sheets and sheet-normal directions because there is still no clear mechanism that is responsible for their production.

The fact that active forces are not conservative in general [25] implies that no potential can be defined for them; and therefore, a strain energy cannot be formulated. And for this reason, in the following, we will not consider this case. Nevertheless, some consistently derived variants have been proposed as active energy functions (see e.g. $[10,15,26]$ ) to model the active biomechanical deformations and/or to study the overall solvability of the mechanical problem in relation with strong ellipticity conditions.

In the active stress model, it is assumed that $\mathbf{P}=\mathbf{P}_{A}+\mathbf{P}_{P}$. Such assumption is widely used for simulations of the electromechanical activity of the heart $[4,9,11]$. Here,

$$
\mathbf{P}_{P}=\psi_{1} \mathbf{F}-p J \mathbf{F}^{-T}+\psi_{4, f} \mathbf{f} \otimes \mathbf{f}_{0}+\psi_{4, s} \mathbf{s} \otimes \mathbf{s}_{0}+\psi_{8, f s}\left(\mathbf{f} \otimes \mathbf{s}_{0}+\mathbf{s} \otimes \mathbf{f}_{0}\right),
$$

and

$$
\psi_{1}=\frac{a}{2} \exp \left(b\left[I_{1}-3\right]\right), \quad \psi_{4, i}=a_{i}\left(I_{4, i}-1\right) \exp \left(b_{i}\left[I_{4, i}-1\right]^{2}\right), \psi_{8, f_{s}}=a_{f_{s}} I_{8, f_{s}} \exp \left(b_{f_{s}} I_{8, f_{s}}^{2}\right) ;
$$

that is, $\mathbf{P}_{P}$ is the passive part of the stress given by $\partial \mathcal{W} / \partial \mathbf{F}$.

The active tension $T_{\mathrm{A}}$ is usually modeled considering the crossbridge dynamics inside the sarcomeres. It will, in general, depend on the calcium concentration available for binding with troponin and on the length of the sarcomeres $[27,28]$. In this study, we consider $T_{\mathrm{A}}$ to be given, and we impose the force in the fibers direction by using the same tensorial component as in the passive law (2.5). Several other models have been proposed in the literature for the tensorial component (a crucial point in the modeling of the electro-mechanical activity of cardiac tissue), but only a few of them address the correct stability conditions to guarantee the existence of solutions, as discussed in [16]. Our choice is intended to allow the preservation of ellipticity conditions of the passive strain energy function.

\subsection{The active strain formulation}

We assume that the deformation gradient $\mathbf{F}$ for the active strain model admits a Lee's multiplicative decomposition [29], that is,

$$
\mathbf{F}=\mathbf{F}_{\mathrm{E}} \mathbf{F}_{\mathrm{A}},
$$

where $\mathbf{F}_{\mathrm{E}}$ is a passive elastic deformation, and $\mathbf{F}_{\mathrm{A}}$ is an active factor to be prescribed (see Figure 2, left). The latter represents at a macroscopic level, the contraction of the sarcomeres depending on the calcium release, electrical excitation, and related phenomena taking place at cell and sub-cell scales.

Similar factorizations of the deformation gradient have been proposed, for instance, in the subject of finite elastoplasticity and applications in growth modeling [29-32] or mechano-chemical
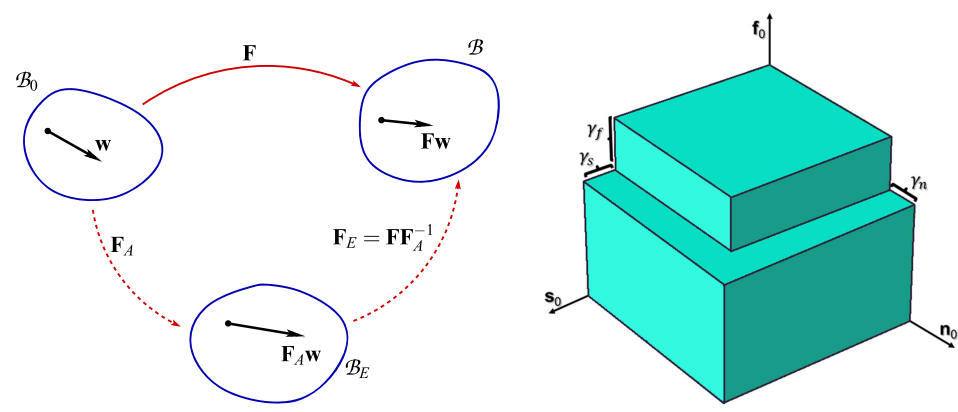

Figure 2. Decomposition of the deformation process under the active strain assumption (left), where $\mathcal{B}_{0}$, $\mathcal{B}_{\mathrm{E}}$ and $\mathcal{B}$ denote a body in its reference, local incompatible intermediate, and current configuration; and exemplification of the deformations at the cell level induced by the active components $\gamma_{f}, \gamma_{n}$, and $\gamma_{s}$ (right). 
interactions [33] and more recently in cardiac electromechanics [12-14]. However, (2.6) is not to be confused with the product of deviatoric and dilational responses $\mathbf{F}=\mathbf{F}_{\mathrm{vol}} \tilde{\mathbf{F}}$, where $\operatorname{det} \tilde{\mathbf{F}}=1$, implying that the deformation induced by $\mathbf{F}_{\mathrm{vol}}$ is (the only one) influencing the changes of volume in the material [31]. Furthermore, $\mathbf{F}$ is given by the gradient of a vector map, whereas $\mathbf{F}_{\mathrm{E}}$ and $\mathbf{F}_{\mathrm{A}}$ are not, in general, because there is no physiological motion corresponding to the shortening of fibers without enforcing muscle contraction.

To construct the active part of the deformation, we start from considerations at the cell level. Defining the variables $\gamma_{f}, \gamma_{s}$ and $\gamma_{n}$ (see Figure 2, right) as the relative displacements in the directions $\mathbf{f}_{0}, \mathbf{s}_{0}, \mathbf{n}_{0}$, (fibers, sheets, and sheets-normal directions) of a single cell, respectively, we write the local deformation as

$$
\mathbf{F}_{\mathrm{A}}=\mathbf{I}+\gamma_{f} \mathbf{f}_{0} \otimes \mathbf{f}_{0}+\gamma_{s} \mathbf{s}_{0} \otimes \mathbf{s}_{0}+\gamma_{n} \mathbf{n}_{0} \otimes \mathbf{n}_{0} .
$$

Its determinant and inverse read

$$
\begin{aligned}
\operatorname{det} \mathbf{F}_{\mathrm{A}} & =\left(1+\gamma_{f}\right)\left(1+\gamma_{s}\right)\left(1+\gamma_{n}\right), \\
\mathbf{F}_{\mathrm{A}}^{-1} & =\mathbf{I}-\frac{\gamma_{f}}{1+\gamma_{f}} \mathbf{f}_{0} \otimes \mathbf{f}_{0}-\frac{\gamma_{s}}{1+\gamma_{s}} \mathbf{s}_{0} \otimes \mathbf{s}_{0}-\frac{\gamma_{n}}{1+\gamma_{n}} \mathbf{n}_{0} \otimes \mathbf{n}_{0} .
\end{aligned}
$$

The factors $\gamma_{i}, i \in\{f, s, n\}$ denote active strain functions that, in the context of the coupling of cardiac elastostatics with electrophysiology, carry the relevant information of the electrical propagation through the tissue and kinematics of its micro-structure.

In [12-14], the contribution of the terms depending on $\gamma_{s}$ and $\gamma_{n}$ is neglected for simplicity. Analogously, for most of the active stress models [9,34], the active tension is assumed to act exclusively along the fibers direction. Here, we opt for the inclusion of the active contributions in the remaining directions but under the assumption that $\gamma_{n}=\gamma_{s}$; that is, we consider transverse isotropy at the microscopic level. Such consideration is motivated by the fact that, at the cell level, there is only one clear preferred direction: the one along which the myocytes contract. The myocyte, however, has still an anisotropic active mechanical response. We stress that this does not correspond to a modeling simplification but to imposing some physiological conditions. In fact, the general orthotropic behavior is due to the sum of the constituents of the cardiac muscle, cells and collagen, among others; the latter not contributing to any substantial active component. These ideas, contained in (2.6), imply a coupling of an orthotropic passive behavior described by (2.2) and a transversely isotropic active contraction at the cell level. Moreover, because intact myocytes, basically made of water, are constrained by the presence of sarcolemma, their motion can be considered isochoric at physiological pressure; that is, their volume can be considered constant [35,36]; and hence, we assume (following also [32]) that

$$
J_{\mathrm{A}}=1,
$$

which, in addition, allows us to express $\gamma_{s}$ as a function of $\gamma_{f}$. Clearly, this condition represents incompressibility only in a formal way, provided that (2.6) is a virtual decomposition. From (2.7) and (2.8), we deduce that $\gamma_{s}=1 / \sqrt{1+\gamma_{f}}-1$; and therefore, the constraint $\gamma_{f}>-1$ is naturally understood. Moreover, if $\gamma_{f}$ is negative, then $\gamma_{s}$ is positive and vice versa. In addition, if $\gamma_{f} \rightarrow \infty$, then $\gamma_{s} \rightarrow-1$, which agrees with the physiological bound $\gamma_{s}>-1$. This behavior is shown in Figure 3 (left). Moreover, because a cardiomyocyte shortens up to a $70 \%$ of its rest length $[37,38]$, we consider the bound $-0.3<\gamma_{f} \leq 0$. Condition (2.8) is not regarded as a constraint associated to the system to be solved, but it is rather a constitutive relation given along with (2.6).

Given that $\gamma_{f}$ represents the coupling between electrophysiology and cardiac elasticity, its evolution can be described via, for example, simple activation models based on ordinary differential equations $[14,28]$; however, for the subsequent elastostatic analysis, as for $T_{\mathrm{A}}$ in the active stress case, we consider it as a space-dependent parameter.

Decomposition (2.6) implies that an intermediate (only virtual) configuration exists between the actual and reference states. In this intermediate state, we define the stored energy function as 

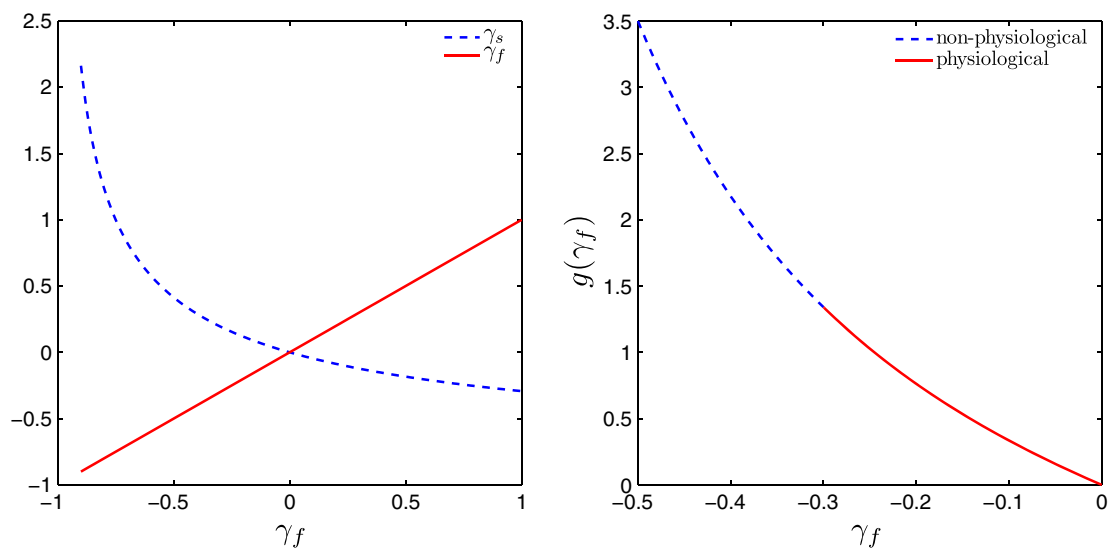

Figure 3. Active strain: behavior of the active strain function $\gamma_{s}$ as a function of $\gamma_{f}$ (left); contribute of the active strain in the direction of the fibers described by the function $g\left(\gamma_{f}\right)$ : the solid line represents the physiologically relevant region (right).

$\widehat{\mathcal{W}}=\mathcal{W}\left(\mathbf{F}_{\mathrm{E}}\right)$, which is then transformed in the reference configuration into the new strain energy function $\mathcal{W}_{\text {strain }}$, able to describe active deformations, through the relation

$$
\mathcal{W}_{\text {strain }}=J_{\mathrm{A}} \widehat{\mathcal{W}}=\mathcal{W}\left(\mathbf{F F}_{\mathrm{A}}^{-1}\right) \text {. }
$$

Even if we assume $J_{\mathrm{A}}=1$, the energies $\mathcal{W}_{\text {strain }}\left(\mathbf{F F}_{\mathrm{A}}^{-1}\right)$ and $\mathcal{W}(\mathbf{F})$ actually differ.

\section{ACTIVE STRESS VERSUS ACTIVE STRAIN FOR DIFFERENT MATERIALS}

For the sake of clarity, in what follows, we omit the terms involving the incompressibility constraint, because they are not relevant to the discussion and can be added at a later stage without loss of generality. Moreover, we keep the same notation for stress tensors under active stress or strain. We underline that the active strain and active stress models actually coincide in the case of infinitesimal deformations [13].

\subsection{Linear dependence on the deformation gradient}

Let us first consider the case of a passively isotropic neo-Hookean material, with energy function

$$
\mathcal{W}=\frac{\mu}{2}\left(I_{1}-3\right)
$$

and passive first Piola-Kirchhoff stress tensor given by

$$
\mathbf{P}_{P}=\mu \mathbf{F},
$$

so that in the active stress formulation we have

$$
\mathbf{P}=\mu \mathbf{F}+T_{\mathrm{A}} \mathbf{f} \otimes \mathbf{f}_{0} .
$$

The energy in the elastic configuration, under active strain, is $\widehat{\mathcal{W}}=\mu / 2\left(I_{1}^{\mathrm{E}}-3\right)$, where $I_{1}^{\mathrm{E}}$ represents the first invariant computed in the intermediate configuration, that is, $I_{1}^{\mathrm{E}}=\operatorname{tr}\left(\mathbf{F E}^{T} \mathbf{F E}\right)$. Then, when applying the active strain decomposition, we pull back the energy from the intermediate to the reference configuration to obtain

$$
\mathcal{W}_{\text {strain }}=\frac{\mu}{2}\left\{\left(1+\gamma_{f}\right) I_{1}-\left(\gamma_{f}+\gamma_{f} \frac{\gamma_{f}+2}{\left(1+\gamma_{f}\right)^{2}}\right) I_{4, f}-3\right\},
$$

where $I_{1}^{\mathrm{E}}$ has been transformed thanks to the formula

$$
I_{1}^{\mathrm{E}}=I_{1}-\gamma_{f} \frac{\gamma_{f}+2}{\left(1+\gamma_{f}\right)^{2}} I_{4, f}-\gamma_{s} \frac{\gamma_{s}+2}{\left(1+\gamma_{s}\right)^{2}} I_{4, s}-\gamma_{n} \frac{\gamma_{n}+2}{\left(1+\gamma_{n}\right)^{2}} I_{4, n},
$$


and the fact that $I_{1}=I_{4, f}+I_{4, s}+I_{4, n}$ and $\gamma_{n}=\gamma_{s}$. Not surprisingly, the energy function has changed, because during active contraction, the material properties of the muscle actually change. Defining

$$
-\gamma_{f}-\gamma_{f} \frac{\gamma_{f}+2}{\left(1+\gamma_{f}\right)^{2}}=: g\left(\gamma_{f}\right)
$$

from (3.4), we can write the stress in the active strain formulation by differentiating the new energy function with respect to the deformation gradient, to obtain

$$
\mathbf{P}=\mu\left(1+\gamma_{f}\right) \mathbf{F}+\mu g\left(\gamma_{f}\right) \mathbf{f} \otimes \mathbf{f}_{0} .
$$

From this perspective, we compare (3.3) and (3.5). First, the active strain approach is consistent with passive models in the sense that imposing $\gamma_{f}=0$, we recover (3.2). Including the active terms, we see that $\mu g\left(\gamma_{f}\right)$ plays a similar role as $T_{\mathrm{A}}$, and its magnitude in the fiber direction is not increasing linearly with $\gamma_{f}$ as depicted in Figure 3 (right). The major difference in both strategies is found in the factor $\left(1+\gamma_{f}\right)$ in front of $\mathbf{F}$, which cannot be retrieved in the active stress formulation, and that can be interpreted as a local change of the material properties in the presence of active contraction. More precisely, this softening of the material obeys to the fact that the first term describes the deformation in all directions, whereas the second term accounts for the deformation in the direction of $\mathbf{f}_{0}$. From this term, we observe, once again, the bound $\gamma_{f}>-1$.

\subsection{Materials described by exponential laws}

Next, we consider the case of an exponential constitutive relation of the form

$$
\mathcal{W}=\frac{a}{2 b} \exp \left(b\left[I_{1}-3\right]\right),
$$

which introduces the nonlinearity of the material but still describes isotropic behavior. The Piola stress in the active stress approach reads

$$
\mathbf{P}=a \exp \left(b\left[I_{1}-3\right]\right) \mathbf{F}+T_{\mathrm{A}} \mathbf{f} \otimes \mathbf{f}_{0},
$$

whereas, for the active strain model, using the same argument as before, we obtain

$$
\mathcal{W}_{\text {strain }}=\frac{a}{2 b} \exp \left(b\left[I_{1}^{\mathrm{E}}-3\right]\right) \text {; }
$$

and therefore,

$$
\mathbf{P}=a \exp \left(b\left[I_{1}^{\mathrm{E}}-3\right]\right)\left(1+\gamma_{f}\right) \mathbf{F}+a \exp \left(b\left[I_{1}^{\mathrm{E}}-3\right]\right) g\left(\gamma_{f}\right) \mathbf{f} \otimes \mathbf{f}_{0} .
$$

The first term in (3.8) represents the behavior of the material in all directions, whereas the second term, responsible for the active contraction in the fibers direction, is not linear anymore with respect to $\mathbf{F}$. In this example, the active behavior and the passive behavior are not split, and their particular action cannot be easily identified. Moreover, there is a mixed dependence on the isotropic and fiber-dependent active deformations given by $I_{1}^{\mathrm{E}}$, which is a linear combination of $I_{1}$ and $I_{4, f}$.

\subsection{Orthotropic Holzapfel-Ogden materials}

Under the active stress formulation, using the orthotropic law (2.2), we obtain

$$
\mathbf{P}=2 \psi_{1} \mathbf{F}+\left(2 \psi_{4, f}+T_{\mathrm{A}}\right) \mathbf{f} \otimes \mathbf{f}_{0}+2 \psi_{4, s} \mathbf{s} \otimes \mathbf{s}_{0}+\psi_{8, f s}\left(\mathbf{f} \otimes \mathbf{s}_{0}+\mathbf{s} \otimes \mathbf{f}_{0}\right) .
$$

To obtain the new energy for the active strain model, we use the relations

$$
I_{4, f}^{\mathrm{E}}=\left(1+\gamma_{f}\right)^{-2} I_{4, f}, \quad I_{4, s}^{\mathrm{E}}=\left(1+\gamma_{f}\right) I_{4, s}, \quad I_{8, f s}^{\mathrm{E}}=\left(1+\gamma_{f}\right)^{-1 / 2} I_{8, f s},
$$

to have

$$
\begin{aligned}
\mathbf{P}= & 2\left(1+\gamma_{f}\right) \psi_{1}^{\mathrm{E}} \mathbf{F}+2\left[\left(1+\gamma_{f}\right)^{-2} \psi_{4, f}^{\mathrm{E}}+g\left(\gamma_{f}\right) \psi_{1}^{\mathrm{E}}\right] \mathbf{f} \otimes \mathbf{f}_{0} \\
& +2\left(1+\gamma_{f}\right) \psi_{4, s}^{\mathrm{E}} \mathbf{s} \otimes \mathbf{s}_{0}+\left(1+\gamma_{f}\right)^{-1 / 2} \psi_{8, f s}^{\mathrm{E}}\left(\mathbf{f} \otimes \mathbf{s}_{0}+\mathbf{s} \otimes \mathbf{f}_{0}\right),
\end{aligned}
$$


where

$$
\psi_{1}^{\mathrm{E}}=\frac{a}{2} \exp \left(b\left[I_{1}^{\mathrm{E}}-3\right]\right), \psi_{4, i}^{\mathrm{E}}=a_{i}\left(I_{4, i}^{\mathrm{E}}-1\right) \exp \left(b_{i}\left[I_{4, i}^{\mathrm{E}}-1\right]^{2}\right), \psi_{8, f_{s}}^{\mathrm{E}}=a_{f_{s}} I_{8, f_{s}}^{\mathrm{E}} \exp \left(b_{f_{s}}\left[I_{8, f_{s}}^{\mathrm{E}}\right]^{2}\right) .
$$

The straightforward transformation of the anisotropic invariants (2.3) suggests that under active strain, the stress tensor will resemble the one obtained with active stress in the fibers and sheets directions. Nonetheless, we expect a different behavior, because the active strain is modifying the contribution of each anisotropic term. In particular, because $\gamma_{f}$ is assumed negative, we expect an increase in the material stiffness in the fibers direction, a softening in the sheets direction, and an increased shear stress (stress in the components involving the two directions). This will be observed numerically in Sections 6.1, 6.6, and 6.7.

\subsection{Strong ellipticity in the active strain and stress formulations}

In mechanical analysis, strict convexity of the energy function may be too restrictive, not allowing the system to reproduce some physical solutions, and it is usual to require other weaker conditions, such as strong ellipticity (see e.g. [10,39]):

$$
\frac{\partial P_{i j}}{\partial F_{p q}} u_{i} u_{p} w_{j} w_{q}>0, \quad \forall\|\boldsymbol{u}\|,\|\boldsymbol{w}\|=1 \text { such that } \mathbf{F}^{-T}: \boldsymbol{u} \otimes \boldsymbol{w}=0 .
$$

We start from the exponential constitutive law (3.6), which is known to satisfy the strong ellipticity condition.

Lemma 3.5

The exponential energy function for active response (3.7) is strongly elliptic for $\gamma_{f} \in(-1, \infty)$.

Proof

Note, first of all, that for $\gamma_{f} \rightarrow-1$ the energy goes to infinity, as we are requiring one dimension to go to zero. So suppose $\gamma_{f} \neq-1$. The condition $J_{\mathrm{A}}>0$ requires also that $\gamma_{f}>-1$. In the active strain model, condition (3.10) directly leads to

$$
\begin{aligned}
& \left(1+\gamma_{f}\right)\left[1+b\left(1+\gamma_{f}\right)(\boldsymbol{u} \cdot \mathbf{F} \boldsymbol{w})^{2}+b g\left(\gamma_{f}\right)(\boldsymbol{u} \cdot \mathbf{F} \boldsymbol{w})\left(\boldsymbol{u} \cdot \mathbf{F f}_{0}\right)\left(\boldsymbol{w} \cdot \mathbf{f}_{0}\right)\right] \\
& \quad+g\left(\gamma_{f}\right)\left[b\left(1+\gamma_{f}\right)(\boldsymbol{u} \cdot \mathbf{F} \boldsymbol{w})\left(\boldsymbol{u} \cdot \mathbf{F f}_{0}\right)\left(\boldsymbol{w} \cdot \mathbf{f}_{0}\right)+b g\left(\gamma_{f}\right)\left(\boldsymbol{u} \cdot \mathbf{F f}_{0}\right)^{2}\left(\boldsymbol{w} \cdot \mathbf{f}_{0}\right)^{2}+\left(\boldsymbol{w} \cdot \mathbf{f}_{0}\right)^{2}\right]>0 .
\end{aligned}
$$

Grouping the arguments in the square brackets, we arrive at

$$
b\left[\left(1+\gamma_{f}\right)(\boldsymbol{u} \cdot \mathbf{F} \boldsymbol{w})+g\left(\gamma_{f}\right)\left(\boldsymbol{u} \cdot \mathbf{F} \mathbf{f}_{0}\right)\left(\boldsymbol{w} \cdot \mathbf{f}_{0}\right)\right]^{2}+\left(1+\gamma_{f}\right)+g\left(\gamma_{f}\right)\left(\boldsymbol{w} \cdot \mathbf{f}_{0}\right)^{2}>0 .
$$

Inequality (3.11) is always valid for contraction (i.e., $-1 \leq \gamma_{f} \leq 0$ ), because all terms are positive. In case of dilation, instead, we have that $\gamma_{f}>0$ and then $g\left(\gamma_{f}\right)$ takes negative values. Recalling the Schwarz inequality $-\left(\boldsymbol{w} \cdot \mathbf{f}_{0}\right)^{2} \geq-\|\boldsymbol{w}\|\left\|\mathbf{f}_{0}\right\|=-1$, it is not difficult to see that if $\gamma_{f}>0$, then

$$
\left(1+\gamma_{f}\right)+g\left(\gamma_{f}\right)\left(\boldsymbol{w} \cdot \mathbf{f}_{0}\right)^{2} \geq\left(1+\gamma_{f}\right)+g\left(\gamma_{f}\right)=\frac{1}{\left(1+\gamma_{f}\right)^{2}}>0
$$

yielding strong ellipticity for all $\gamma_{f}>-1$.

Lemma 3.6

Under the active stress assumption, the strong ellipticity is always satisfied for muscle contraction $\left(T_{\mathrm{A}} \geq 0\right)$. In case of dilation $\left(T_{\mathrm{A}}<0\right)$, strong ellipticity holds for

$$
-T_{\mathrm{A}}<a \exp \left(b\left[I_{1}-3\right]\right)\left[b(\boldsymbol{u} \cdot \mathbf{F} \boldsymbol{w})^{2}+1\right] .
$$

Proof

Concerning the active stress model, condition (3.10) leads to

$$
a \exp \left(b\left[I_{1}-3\right]\right)\left[b(\boldsymbol{u} \cdot \mathbf{F} \boldsymbol{w})^{2}+1\right]+T_{\mathrm{A}}\left(\boldsymbol{w} \cdot \mathbf{f}_{0}\right)^{2}>0,
$$


which holds for all non-negative values of $T_{\mathrm{A}}$. The bound for $T_{\mathrm{A}}<0$ follows directly from Schwarz inequality.

In our specific problem, we do not focus on active elongation, because dilation is mainly due to the passive response of the material subject to the blood pressure entering the ventricles. Nonetheless, we observe that the active strain formulation is able to preserve the strong ellipticity condition of the passive constitutive law (see also [16] for a more general proof), whereas the active stress model presents some constraints. With the example presented earlier, we show that the active stress model introduces effects that can modify the original properties of the passive material constitutive law; and therefore, the tensorial form of the active stress tensor must be chosen carefully to allow a considerable range of deformations. As in [5], an orthotropic active stress may be considered by imposing dilation in the sheets and sheets-normal directions.

Similar results hold for neo-Hookean materials, imposing $b=0$ in (3.11), (3.12), and identifying $a$ with $\mu$. Let us now consider the orthotropic law (2.2), which does not fulfill the strong ellipticity condition [20]. First, we readily see that the active strain and active stress formulations modify the inequality to be verified.

In the active strain model, the same considerations as before are valid for the isotropic term, and we only need to examine the anisotropic part of the energy function. After differentiating, we find that the following conditions should hold

$$
\begin{gathered}
{\left[\frac{1}{\left(1+\gamma_{f}\right)^{2}}+2 b_{f}\left(I_{4, f}^{\mathrm{E}}-1\right)^{2}\right]\left(\boldsymbol{u} \cdot \mathbf{F f}_{0}\right)^{2}+\left[\frac{I_{4, f}}{\left(1+\gamma_{f}\right)^{2}}-1\right]>0,} \\
{\left[\left(1+\gamma_{f}\right)+2 b_{s}\left(I_{4, s}^{\mathrm{E}}-1\right)^{2}\right]\left(\boldsymbol{u} \cdot \mathbf{F s}_{0}\right)^{2}+\left[I_{4, s}\left(1+\gamma_{f}\right)-1\right]>0 .}
\end{gathered}
$$

If we consider, for a moment, only passive behavior (i.e. $\gamma_{f}=0$ ), the first inequality in (3.13) is not satisfied in general, in the case of compression $\left(I_{4, f}<1\right)$. However, it turns out that the active strain formulation reverses this condition: because $\gamma_{f}$ is negative during active contraction, the quantity $I_{4, f} /\left(1+\gamma_{f}\right)^{2}-1$ will be positive (if no passive compression is imposed). On the other hand, the term $I_{4, s}\left(1+\gamma_{f}\right)-1$ will assume negative values. In this sense, the active strain formulation 'regularizes' the passive law in the direction $\mathbf{f}_{0}$ and 'penalizes' it in the direction $\mathbf{s}_{0}$. Regarding the last term of the energy function (2.2), we find that

$$
\left[1+2 b_{f_{S}}\left(I_{8, f s}^{\mathrm{E}}\right)^{2}\right]\left[\left(\boldsymbol{u} \cdot \mathbf{F} \mathbf{f}_{0}\right)\left(\boldsymbol{w} \cdot \mathbf{s}_{0}\right)+\left(\boldsymbol{u} \cdot \mathbf{F} \mathbf{s}_{0}\right)\left(\boldsymbol{w} \cdot \mathbf{f}_{0}\right)\right]^{2}+I_{8, f_{S}}\left(\boldsymbol{w} \cdot \mathbf{f}_{0}\right)\left(\boldsymbol{w} \cdot \mathbf{s}_{0}\right)>0 .
$$

The active stress model acts only in the fiber direction; and hence, the same conditions of the passive law hold for the terms depending on $I_{1}, I_{4, s}$ and $I_{8, f_{s}}$. Concerning the fiber terms, we obtain

$$
2 a_{f} \exp \left(b_{f}\left[I_{4, f}-1\right]^{2}\right)\left\{\left[1+2 b_{f}\left(I_{4, f}-1\right)^{2}\right]\left(\boldsymbol{u} \cdot \mathbf{F f}_{0}\right)+\left(I_{4, f}-1\right)\right\}+T_{\mathrm{A}}>0 .
$$

The active tension $T_{\mathrm{A}}$ is positive under active contraction, which entails a 'regularization' of the energy in the fiber direction. This helps the fulfillment of (3.14).

In summary, by acting differently on (3.10), both models are able to regularize the fibers-related part of the energy function. Nevertheless, in the full orthotropic case, strong ellipticity is not guaranteed in general.

\section{CONSISTENT LINEARIZATION AND WELL-POSEDNESS ANALYSIS}

We introduce the following linearized problem from (2.4) (by assuming homogeneous Dirichlet data in, at least, a small region of the boundary), considered around a generic state $(\hat{\boldsymbol{u}}, \hat{p})$ : Find $\boldsymbol{u} \in V, p \in Q$ such that

$$
\begin{array}{r}
\int_{\mathcal{B}_{0}}\left(\frac{\partial \mathbf{P}(\hat{\boldsymbol{u}}, \hat{p})}{\partial \mathbf{F}}: \nabla \boldsymbol{u}\right): \nabla \boldsymbol{v}+\int_{\mathcal{B}_{0}} p \frac{\partial \mathbf{P}(\hat{\boldsymbol{u}}, \hat{p})}{\partial p}: \nabla \boldsymbol{v}=R_{\boldsymbol{u}}(\hat{\boldsymbol{u}}, \hat{p}, \boldsymbol{v}) \\
\int_{\mathcal{B}_{0}} q \hat{J} \hat{\mathbf{F}}^{-T}: \nabla \boldsymbol{u}=R_{p}(\hat{\boldsymbol{u}}, q),
\end{array}
$$


for all $v \in V, q \in Q$, where $R_{\boldsymbol{u}}$ and $R_{p}$ are

$$
R_{\boldsymbol{u}}(\hat{\boldsymbol{u}}, \hat{p}, \boldsymbol{w})=\int_{\mathcal{B}_{0}} \boldsymbol{\ell} \cdot \boldsymbol{v}-\int_{\mathcal{B}_{0}} \hat{\mathbf{P}}: \nabla \boldsymbol{v}, \quad R_{p}(\hat{\boldsymbol{u}}, q)=\int_{\mathcal{B}_{0}} q(\hat{J}-1) .
$$

Let us consider the active strain model first. Using (3.9) in the first equation of (4.1) and expanding the derivatives, we arrive at

$$
A_{\mathrm{iso}}+A_{\mathrm{iso}, f}+A_{f}+A_{s}+A_{f, s}+A_{p}=R_{\boldsymbol{u}}(\hat{\boldsymbol{u}}, \hat{p}, \boldsymbol{w}),
$$

where

$$
\begin{aligned}
& A_{\text {iso }}=\int_{\mathcal{B}_{0}} 4 b \hat{\psi}_{1}^{\mathrm{E}}\left(1+\gamma_{f}\right)\left[\left(1+\gamma_{f}\right) \hat{\mathbf{F}}: \nabla \boldsymbol{u}+g\left(\gamma_{f}\right) \hat{\mathbf{f}} \cdot \nabla \boldsymbol{u} \mathbf{f}_{0}\right] \hat{\mathbf{F}}: \nabla \boldsymbol{w}+2\left(1+\gamma_{f}\right) \hat{\psi}_{1}^{\mathrm{E}} \nabla \boldsymbol{u}: \nabla \boldsymbol{w} \\
& A_{\text {iso }, f}=\int_{\mathcal{B}_{0}} 4 b \psi_{1}^{\mathrm{E}} g\left(\gamma_{f}\right)\left[\left(1+\gamma_{f}\right) \hat{\mathbf{F}}: \nabla \boldsymbol{u}+g\left(\gamma_{f}\right) \hat{\mathbf{f}} \cdot \nabla \boldsymbol{u} \mathbf{f}_{0}\right] \hat{\mathbf{f}} \cdot \nabla \boldsymbol{w} \mathbf{f}_{0}+2 g\left(\gamma_{f}\right) \hat{\psi}_{1}^{\mathrm{E}} \nabla \boldsymbol{u} \mathbf{f}_{0} \cdot \nabla \boldsymbol{w} \mathbf{f}_{0} \\
& A_{f}=\int_{\mathcal{B}_{0}} \frac{4 b_{f}\left(\hat{I}_{4, f}^{\mathrm{E}}-1\right) \hat{\psi}_{4, f}^{\mathrm{E}}+2 a_{f} \exp \left(b_{f}\left[\hat{I}_{4, f}^{\mathrm{E}}-1\right]^{2}\right)}{\left(1+\gamma_{f}\right)^{4}}\left(\hat{\mathbf{f}} \cdot \nabla \boldsymbol{u} \mathbf{f}_{0}\right)\left(\hat{\mathbf{f}} \cdot \nabla \boldsymbol{w} \mathbf{f}_{0}\right) \\
& +\frac{2 \hat{\psi}_{4, f}^{\mathrm{E}}}{\left(1+\gamma_{f}\right)^{2}} \nabla \boldsymbol{u} \mathbf{f}_{0} \cdot \nabla \boldsymbol{w} \mathbf{f}_{0} \\
& A_{s}=\int_{\mathcal{B}_{0}} \frac{4 b_{s}\left(\hat{I}_{4, s}^{\mathrm{E}}-1\right) \psi_{4, s}^{\mathrm{E}}+2 a_{s} \exp \left(b_{s}\left[\hat{I}_{4, s}^{\mathrm{E}}-1\right]^{2}\right)}{\left(1+\gamma_{f}\right)^{-2}}\left(\hat{\mathbf{s}} \cdot \nabla \boldsymbol{u} \mathbf{s}_{0}\right)\left(\hat{\mathbf{s}} \cdot \nabla \boldsymbol{w} \mathbf{s}_{0}\right) \\
& +\frac{2 \hat{\psi}_{4, s}^{\mathrm{E}}}{\left(1+\gamma_{f}\right)^{-1}} \nabla \boldsymbol{u} \mathbf{s}_{0} \cdot \nabla \boldsymbol{w} \mathbf{s}_{0} \\
& A_{p}=-\int_{\mathcal{B}_{0}} \hat{p} \hat{J}\left(\hat{\mathbf{F}}^{-T}: \nabla \boldsymbol{u}\right)\left(\hat{\mathbf{F}}^{-T}: \nabla \boldsymbol{w}\right)+\int_{\mathcal{B}_{0}} \hat{p} \hat{J} \hat{\mathbf{F}}^{-T} \nabla \boldsymbol{u}^{T}: \nabla \boldsymbol{w} \hat{\mathbf{F}}^{-1}-\int_{\mathcal{B}_{0}} p \hat{J} \hat{\mathbf{F}}^{-T}: \nabla \boldsymbol{w} \\
& A_{f, s}=\int_{\mathcal{B}_{0}} \frac{2 b_{f s} \hat{I}_{8, f s}^{\mathrm{E}} \hat{\psi}_{8, f_{s}}^{\mathrm{E}}+a_{f s} \exp \left(b_{f_{s}} \hat{I}_{8, f s}^{2, E}\right)}{1+\gamma_{f}}\left(\hat{\mathbf{f}} \cdot \nabla \boldsymbol{u} \mathbf{s}_{0}+\hat{\mathbf{s}} \cdot \nabla \boldsymbol{u} \mathbf{f}_{0}\right)\left(\hat{\mathbf{f}} \cdot \nabla \boldsymbol{w} \mathbf{s}_{0}+\hat{\mathbf{s}} \cdot \nabla \boldsymbol{w} \mathbf{f}_{0}\right) \\
& +\int_{\mathcal{B}_{0}} \frac{\hat{\psi}_{8, f_{s}}^{\mathrm{E}}}{\sqrt{1+\gamma_{f}}}\left(\nabla \boldsymbol{u} \mathbf{f}_{0} \cdot \nabla \boldsymbol{w} \mathbf{s}_{0}+\nabla \boldsymbol{u s} \cdot \nabla \boldsymbol{w} \mathbf{f}_{0}\right) .
\end{aligned}
$$

We define two bilinear forms $a(\cdot, \cdot)$ and $b(\cdot, \cdot)$ by

$$
a(\boldsymbol{u}, \boldsymbol{w}):=A_{\mathrm{iso}}+A_{\mathrm{iso}, f}+A_{f}+A_{s}+A_{f, s}, \quad b(\boldsymbol{w}, q):=A_{p},
$$

and we readily notice that $a(\cdot, \cdot)$ is symmetric. Equations (4.1) can be recast in the mixed form: Given $(\hat{\boldsymbol{u}}, \hat{p})$, find $\boldsymbol{u} \in V, p \in Q$ such that

$$
\begin{aligned}
a(\boldsymbol{u}, \boldsymbol{v})+b(\boldsymbol{v}, p) & =R_{\boldsymbol{u}}(\hat{\boldsymbol{u}}, \hat{p}, \boldsymbol{w}) \quad \forall \boldsymbol{v} \in V, \\
b(\boldsymbol{u}, q) & =R_{p}(\hat{\boldsymbol{u}}, q) \quad \forall q \in Q .
\end{aligned}
$$


Because (4.2) holds for any state, in particular, it does for $(\hat{\boldsymbol{u}}, \hat{p})=(\mathbf{0}, 0)$, and assuming $\ell=0$ yields

$$
\begin{aligned}
2 a b & \int_{\mathcal{B}_{0}}\left(1+\gamma_{f}\right) \exp \left(b \gamma_{f}^{2} \frac{2 \gamma_{f}-3}{\left(1+\gamma_{f}\right)^{2}}\right)\left[\left(1+\gamma_{f}\right) \operatorname{div} \boldsymbol{u}+g\left(\gamma_{f}\right) \mathbf{f}_{0} \cdot \nabla \boldsymbol{u} \mathbf{f}_{0}\right] \operatorname{div} \boldsymbol{w} \\
& +a \int_{\mathcal{B}_{0}}\left(1+\gamma_{f}\right) \exp \left(b \gamma_{f}^{2} \frac{2 \gamma_{f}-3}{\left(1+\gamma_{f}\right)^{2}}\right) \nabla \boldsymbol{u}: \nabla \boldsymbol{w} \\
& +a \int_{\mathcal{B}_{0}} g\left(\gamma_{f}\right) \exp \left(b \gamma_{f}^{2} \frac{2 \gamma_{f}-3}{\left(1+\gamma_{f}\right)^{2}}\right) \nabla \boldsymbol{u} \mathbf{f}_{0} \cdot \nabla \boldsymbol{w} \mathbf{f}_{0} \\
& +2 a b \int_{\mathcal{B}_{0}} \exp \left(b \gamma_{f}^{2} \frac{2 \gamma_{f}-3}{\left(1+\gamma_{f}\right)^{2}}\right) g\left(\gamma_{f}\right)\left[\left(1+\gamma_{f}\right) \operatorname{div} \boldsymbol{u}+g\left(\gamma_{f}\right) \mathbf{f}_{0} \cdot \nabla \boldsymbol{u} \mathbf{f}_{0}\right] \mathbf{f}_{0} \cdot \nabla \boldsymbol{w} \mathbf{f}_{0} \\
& +\int_{\mathcal{B}_{0}}\left(4 a_{f} b_{f}\left[\gamma_{f}+g\left(\gamma_{f}\right)\right]^{2}+2 a_{f}\right)\left(1+\gamma_{f}\right)^{-4} \\
& \times \exp \left(b_{f}\left[\gamma_{f}+g\left(\gamma_{f}\right)\right]^{2}\right)\left(\mathbf{f}_{0} \cdot \nabla \boldsymbol{u} \mathbf{f}_{0}\right)\left(\mathbf{f}_{0} \cdot \nabla \boldsymbol{w} \mathbf{f}_{0}\right) \\
& +\int_{\mathcal{B}_{0}} \frac{2 a_{f}\left[\gamma_{f}+g\left(\gamma_{f}\right)\right] \exp \left(b_{f}\left[\gamma_{f}+g\left(\gamma_{f}\right)\right]^{2}\right)}{\left(1+\gamma_{f}\right)^{2}} \nabla \boldsymbol{u} \mathbf{f}_{0} \cdot \nabla \boldsymbol{w} \mathbf{f}_{0}-\int_{\mathcal{B}_{0}} p \operatorname{div} \boldsymbol{w} \\
& +\int_{\mathcal{B}_{0}} \frac{\left(4 a_{s} b_{s} \gamma_{f}^{2}+2 a_{s}\right)}{\left(1+\gamma_{f}\right)^{-2}} \exp \left(b_{s} \gamma_{f}^{2}\right)\left(\mathbf{s}_{0} \cdot \nabla \boldsymbol{u} \mathbf{s}_{0}\right)\left(\mathbf{s}_{0} \cdot \nabla \boldsymbol{w} \mathbf{s}_{0}\right)+\frac{2 a_{s} \gamma_{f} \exp \left(b_{s} \gamma_{f}^{2}\right)}{\left(1+\gamma_{f}\right)^{-1}} \nabla \boldsymbol{u} \mathbf{s}_{0} \cdot \nabla \boldsymbol{w} \mathbf{s}_{0} \\
& +\int_{\mathcal{B}_{0}} \frac{a_{f s}}{1+\gamma_{f}}\left(\mathbf{f}_{0} \cdot \nabla \boldsymbol{u} \mathbf{s}_{0}+\mathbf{s}_{0} \cdot \nabla \boldsymbol{u} \mathbf{f}_{0}\right)\left(\mathbf{f}_{0} \cdot \nabla \boldsymbol{w} \mathbf{s}_{0}+\mathbf{s}_{0} \cdot \nabla \boldsymbol{w} \mathbf{f}_{0}\right)=R_{\boldsymbol{u}}(\mathbf{0}, 0, \boldsymbol{w}),
\end{aligned}
$$

for all $\boldsymbol{w} \in V$, and

$$
\int_{\mathcal{B}_{0}} q \operatorname{div} \boldsymbol{u}=0, \text { for all } q \in Q
$$

Hence, we have the problem: Given $(\hat{\boldsymbol{u}}, \hat{p})=(\mathbf{0}, 0)$, find $\boldsymbol{u} \in V, p \in Q$ such that

$$
\begin{array}{rlrl}
a(\boldsymbol{u}, \boldsymbol{v})+b(\boldsymbol{v}, p) & =R_{\boldsymbol{u}}(\mathbf{0}, 0, \boldsymbol{w}) & \forall \boldsymbol{v} \in V, \\
b(\boldsymbol{u}, q) & =0 & & \forall q \in Q .
\end{array}
$$

\section{Proposition 4.1}

Problem (4.3) is well-posed and admits a unique solution for every $\gamma_{f}$ in the physiological range.

Proof

This system assumes the form of a typical saddle-point problem where the bilinear form $a(\cdot, \cdot)$ is symmetric. From classical results (see e.g. [17]), it is known that the conditions for well-posedness of (4.3) are continuity of $a(\cdot, \cdot)$ in $V$, coercivity of $a(\cdot, \cdot)$ in $V_{\text {div }}=\{\boldsymbol{w} \in V: \operatorname{div} \boldsymbol{w}=0\}$, and the fulfillment of the inf-sup condition for the bilinear form $b(\cdot, \cdot)$ and the spaces $V, Q$ :

$$
\inf _{q \in Q} \sup _{\boldsymbol{v} \in V} \frac{b(\boldsymbol{v}, q)}{\|\boldsymbol{v}\| q \|} \geq C,
$$

for some constant $C>0$. Because in our case $b(\cdot, \cdot)$ is the usual bilinear form associated to the divergence operator, this condition is known to be satisfied. To prove continuity of $a(\cdot, \cdot)$, recall that for $\boldsymbol{v} \in V$, $\|\operatorname{div} \boldsymbol{v}\|_{L^{2}\left(\beta_{0}\right)} \leq\|\nabla \boldsymbol{v}\|_{L^{2}\left(\mathcal{B}_{0}\right)}$. Because $\mathbf{f}_{0}$ is a unit vector, it holds $\left\|\mathbf{f}_{0} \cdot \nabla \boldsymbol{v} \mathbf{f}_{0}\right\|_{L^{2}\left(\mathcal{B}_{0}\right)} \leq$ $K_{1}\|\nabla \boldsymbol{v}\|_{L^{2}\left(\beta_{0}\right)}$ and $\left\|\nabla \boldsymbol{v} \mathbf{f}_{0}\right\|_{L^{2}\left(\beta_{0}\right)} \leq K_{2} \mid\|\nabla \boldsymbol{v}\|_{L^{2}\left(\beta_{0}\right)}$, for some constants $K_{1}$ and $K_{2}$. Similar results hold for the terms involving the direction $\mathbf{s}_{0}$. In the end, the functions depending on $\gamma_{f}$ appearing in the integrals are always positive and finite for physiological regimes $\left(-0.3 \leq \gamma_{f} \leq 0\right)$. These arguments lead to

$$
|a(\boldsymbol{u}, \boldsymbol{w})| \leq M\left(\gamma_{f}\right)\|\boldsymbol{u}\|_{\boldsymbol{H}^{1}\left(\mathcal{B}_{0}\right)}\|\boldsymbol{w}\|_{\boldsymbol{H}^{1}\left(\mathcal{B}_{0}\right)} \quad \forall \boldsymbol{u}, \boldsymbol{w} \in \boldsymbol{H}^{1}\left(\mathcal{B}_{0}\right),
$$


where we indicated the dependence of the continuity constant $M$ on $\gamma_{f}$. In order to prove the coercivity bound, it suffices to note that all terms in the bilinear form are positive.

Repeating the same argument for the active stress formulation implies that for $(\hat{\boldsymbol{u}}, \hat{p})=(\mathbf{0}, 0)$, equations (4.1) become

$$
\begin{aligned}
\bar{a}(\boldsymbol{u}, \boldsymbol{v})+\bar{b}(\boldsymbol{v}, p) & =\bar{F}(\boldsymbol{v}) \quad \forall \boldsymbol{v} \in V, \\
\bar{b}(\boldsymbol{u}, q) & =0 \quad \forall q \in Q,
\end{aligned}
$$

where

$$
\begin{aligned}
\bar{a}(\boldsymbol{u}, \boldsymbol{w}):= & 2 a b \int_{\mathcal{B}_{0}} \operatorname{div} \boldsymbol{u} \operatorname{div} \boldsymbol{w}+a \int_{\mathcal{B}_{0}} \nabla \boldsymbol{u}: \nabla \boldsymbol{w}+4 a_{s} \int_{\mathcal{B}_{0}}\left(\mathbf{s}_{0} \otimes \mathbf{s}_{0}: \nabla \boldsymbol{u}\right)\left(\mathbf{s}_{0} \otimes \mathbf{s}_{0}: \nabla \boldsymbol{w}\right) \\
& +\left(4 a_{f}+T_{\mathrm{A}}\right) \int_{\mathcal{B}_{0}}\left(\mathbf{f}_{0} \otimes \mathbf{f}_{0}: \nabla \boldsymbol{u}\right)\left(\mathbf{f}_{0} \otimes \mathbf{f}_{0}: \nabla \boldsymbol{w}\right) \\
& +2 a_{f s} \int_{\mathcal{B}_{0}}\left[\left(\mathbf{s}_{0} \otimes \mathbf{f}_{0}+\mathbf{f}_{0} \otimes \mathbf{s}_{0}\right): \nabla \boldsymbol{u}\right]\left[\left(\mathbf{s}_{0} \otimes \mathbf{f}_{0}+\mathbf{f}_{0} \otimes \mathbf{s}_{0}\right): \nabla \boldsymbol{w}\right] \\
\bar{b}(\boldsymbol{w}, q):=- & \int_{\mathcal{B}_{0}} q \operatorname{div} \boldsymbol{w}, \quad \bar{F}(\boldsymbol{w}):=-a \int_{\mathcal{B}_{0}} \operatorname{div} \boldsymbol{w}-T_{\mathrm{A}} \int_{\mathcal{B}_{0}}\left(\mathbf{f}_{0} \otimes \mathbf{f}_{0}: \nabla \boldsymbol{w}\right),
\end{aligned}
$$

for all $w \in V, q \in Q$. Analogously to the active strain case, we obtain

$$
|\bar{a}(\boldsymbol{u}, \boldsymbol{w})| \leq\left(2 a b+a+T_{\mathrm{A}}+4 a_{f}+4 a_{s}+4 a_{f s}\right)\|\boldsymbol{u}\|_{\boldsymbol{H}^{1}\left(\mathcal{B}_{0}\right)}\|\boldsymbol{w}\|_{\boldsymbol{H}^{1}\left(\mathcal{B}_{0}\right)},
$$

for all $\boldsymbol{u}, \boldsymbol{w} \in V$; and finally, the positivity of all terms yields the coercivity of $\bar{a}(\cdot, \cdot)$ in $\boldsymbol{H}_{\text {div }}^{\mathbf{1}}\left(\mathcal{B}_{0}\right)$.

\section{DISCRETIZATION BY FINITE ELEMENTS}

Introducing the finite dimensional spaces $V^{h} \subset V$, $\operatorname{dim} V^{h}=N_{h}$, and $Q^{h} \subset Q, \operatorname{dim} Q^{h}=M_{h}$, for the approximation of displacement and pressure, respectively, we write the Galerkin problem associated to (4.2) as follows: Find $\boldsymbol{u}^{h} \in V^{h}, p^{h} \in Q^{h}$ such that

$$
\begin{aligned}
a\left(\boldsymbol{u}^{h}, \boldsymbol{v}^{h}\right)+b\left(\boldsymbol{v}^{h}, p^{h}\right) & =F\left(\boldsymbol{v}^{h}\right) & & \forall \boldsymbol{v}^{h} \in V^{h}, \\
b\left(\boldsymbol{u}^{h}, q^{h}\right) & =G\left(q^{h}\right) & & \forall q^{h} \in Q^{h} .
\end{aligned}
$$

We use for displacements piecewise $d$-linear elements enriched with cubic bubble functions, or alternatively, piecewise $d$-quadratic elements for displacements, and piecewise $d$-linear elements for pressure (giving rise to MINI and Taylor-Hood elements, that is, $\mathbb{P}_{1}^{b}-\mathbb{P}_{1}$ and $\mathbb{P}_{2}-\mathbb{P}_{1}$, respectively). These finite element pairs are known to satisfy the discrete inf-sup condition and perform fairly robustly for linear elasticity problems, as well as for large deformation analysis [19].

\subsection{Newton method}

We state the following Newton scheme for (4.2)

$$
\begin{aligned}
a_{k}\left(\boldsymbol{u}_{k+1}, \boldsymbol{v}\right)+b_{k}\left(\boldsymbol{v}, p_{k+1}\right) & =F_{k}(\boldsymbol{v}) \quad \\
b_{k}\left(\boldsymbol{u}_{k+1}, q\right) & =G_{k}(q) \quad \forall q \in V,
\end{aligned}
$$

where the notation $a_{k}(\cdot, \cdot), b_{k}(\cdot, \cdot), F_{k}(\cdot)$, and $G_{k}(\cdot)$ emphasizes a direct dependence on the solution at Newton step $k$. Using the abridged notation $\mathbf{P}_{k}=\mathbf{P}\left(\boldsymbol{u}_{k}, p_{k}\right)$, we have

$$
a_{k}\left(\boldsymbol{u}_{k+1}, \boldsymbol{v}\right)=\int_{\mathcal{B}_{0}}\left(\frac{\partial \mathbf{P}_{k}}{\partial \mathbf{F}}: \nabla \boldsymbol{u}_{k+1}\right): \nabla \boldsymbol{v}, \quad b_{k}\left(\boldsymbol{u}_{k+1}, q\right)=\int_{\mathcal{B}_{0}} q J_{k} \mathbf{F}_{k}^{-T}: \nabla \boldsymbol{u}_{k+1} .
$$


Let $\left\{\phi^{l}\right\}_{l=1}^{N_{h}}$ and $\left\{\zeta^{n}\right\}_{n=1}^{M_{h}}$ denote the basis for the spaces $V^{h}$ and $Q^{h}$, respectively. Then, at every Newton iteration, our problem reads

$$
\begin{aligned}
u_{k+1}^{m} a_{k}\left(\phi^{m}, \phi^{l}\right)+p_{k+1}^{n}\left(\phi^{l}, \zeta^{n}\right) & =F_{k}\left(\phi^{l}\right) \quad l=1, \ldots, N_{h}, \\
u_{k+1}^{m} b_{k}\left(\phi^{m}, \zeta^{n}\right) & =G_{k}\left(\zeta^{n}\right) \quad n=1, \ldots, M_{h},
\end{aligned}
$$

where the convention of the sum for the repeated indexes applies. In matrix form, we obtain

$$
\left(\begin{array}{cc}
\mathbb{A}_{k} & \mathbb{B}_{k}^{T} \\
\mathbb{B}_{k} & 0
\end{array}\right)\left(\begin{array}{l}
\mathbb{U}_{k+1} \\
\mathbb{P}_{k+1}
\end{array}\right)=\left(\begin{array}{c}
\mathbb{F}_{k} \\
\mathbb{G}_{k}
\end{array}\right)
$$

where the matrices $\mathbb{A}_{k}$ and $\mathbb{B}_{k}$ are defined as $A_{k}^{l m}=a_{k}\left(\phi^{m}, \phi^{l}\right)$ and $B_{k}^{m n}=b_{k}\left(\phi^{m}, \zeta^{n}\right)$. The vector $\mathbb{F}_{k}$ includes the contribution due to the boundary conditions, whereas for $\mathbb{G}_{k}$, we have $G_{k}^{n}=\int_{\mathcal{B}_{0}} \zeta^{n}\left(1-J_{k}\right)$, with $J_{k}=\operatorname{det} \mathbf{F}\left(\boldsymbol{u}_{k}\right)$. Defining the total relative residual as

$$
\varepsilon_{k}=\frac{\left\|\boldsymbol{u}_{k}-\boldsymbol{u}_{k-1}\right\|_{H^{1}}}{\left\|\boldsymbol{u}_{k}\right\|_{H^{1}}}+\frac{\left\|p_{k}-p_{k-1}\right\|_{L^{2}}}{\left\|p_{k}\right\|_{L^{2}}},
$$

we use the stopping criteria $\varepsilon_{k}<\epsilon$, with a given tolerance $\epsilon$; and at each Newton iteration, we solve (5.1) with the UMFPACK method [40] for the two-dimentional examples and the MUMPS routines [41] for examples in three dimensions.

\subsection{The homotopy argument}

Even though the linear problems from Section 4 admit unique solutions, the associated Newton schemes may not converge for any allowed value of $\gamma_{f}$ or $T_{\mathrm{A}}$. As a matter of fact, for large deformation problems, such initial guess is far from the actual solution. A remedy consists in moving smoothly from $(\mathbf{0}, 0)$ to the desired state. A Newton method combined with an incremental step method, or homotopy argument (see e.g. [42]) will be used. We show the development for the active strain case, but analogous arguments apply to the active stress approach.

First, we make explicit in (4.1) the dependence on the quantity to increment (in this case, the active strain $\gamma_{f}$ ). The initial value of $\gamma_{f}$ should be small enough to guarantee the convergence of the Newton method; that is, the deformed configuration should be close enough to $\left(\boldsymbol{u}_{0}^{h}, p_{0}^{h}\right)$. Then, the new solution $\left(\boldsymbol{u}_{k+1}^{h}, p_{k+1}^{h}\right)$ represents the updated initial state that can be used for the next Newton procedure

$$
\begin{aligned}
\hat{a}_{k}^{\gamma_{f}+\Delta \gamma_{f}}\left(\boldsymbol{u}_{k+1}^{h}, \boldsymbol{v}^{h}\right)+b_{k}\left(\boldsymbol{v}^{h}, p_{k+1}^{h}\right) & =\hat{F}_{k}^{\gamma_{f}+\Delta \gamma_{f}}\left(\boldsymbol{v}^{h}\right) & & \forall \boldsymbol{v}^{h} \in V^{h}, \\
b_{k}\left(\boldsymbol{u}_{k+1}^{h}, q^{h}\right) & =G_{k}\left(q^{h}\right) & & \forall q^{h} \in Q^{h},
\end{aligned}
$$

where $\Delta \gamma_{f}$ represents an increment of the active force and $\hat{a}_{k}^{\gamma_{f}+\Delta \gamma_{f}}(\cdot, \cdot), \hat{F}_{k}^{\gamma_{f}+\Delta \gamma_{f}}(\cdot)$ stand for the bilinear and linear forms computed with incremented activation $\gamma_{f}+\Delta \gamma_{f}$. For the convergence of this procedure to be ensured, this $\Delta \gamma_{f}$ should be taken such that the configuration at $\left(\boldsymbol{u}_{k+1}^{h}, p_{k+1}^{h}\right)$ represents a small deformation with respect to that at $\left(\boldsymbol{u}_{k}^{h}, p_{k}^{h}\right)$. Note that the initial value of the activation may depend on different factors (geometry, constitutive law, etc). For instance, a linear material could not require this kind of procedure, whereas a Holzapfel-Ogden material may need a large number of iterations.

In practice, some cases will require to start with small forces and perform a check on the relative residual at the first Newton iteration. More specifically, given $\gamma_{f}$ (or $T_{\mathrm{A}}$ ), its increment $\Delta \gamma_{f}$ (or $\Delta T_{A}$ ), and the Newton and homotopy tolerances $\epsilon, \tau$, we follow Algorithm 1 in the succeeding texts. As it will be clear from the numerical tests presented in Section 6, this algorithm performs quite well in the active strain formulation, whereas it may require several hundreds incremental iterations for the active stress model. To avoid this, we modify slightly the algorithm requiring $\tau_{\min }<\varepsilon_{1}<\tau_{\max }$. If $\varepsilon_{1}>\tau_{\max }$, we half the increment; and if $\varepsilon_{1}<\tau_{\min }$, we double it. Special care must be taken when choosing the interval $\left[\tau_{\min }, \tau_{\max }\right]$; otherwise, the residual may jump above and below the chosen tolerances without entering the second iteration. 


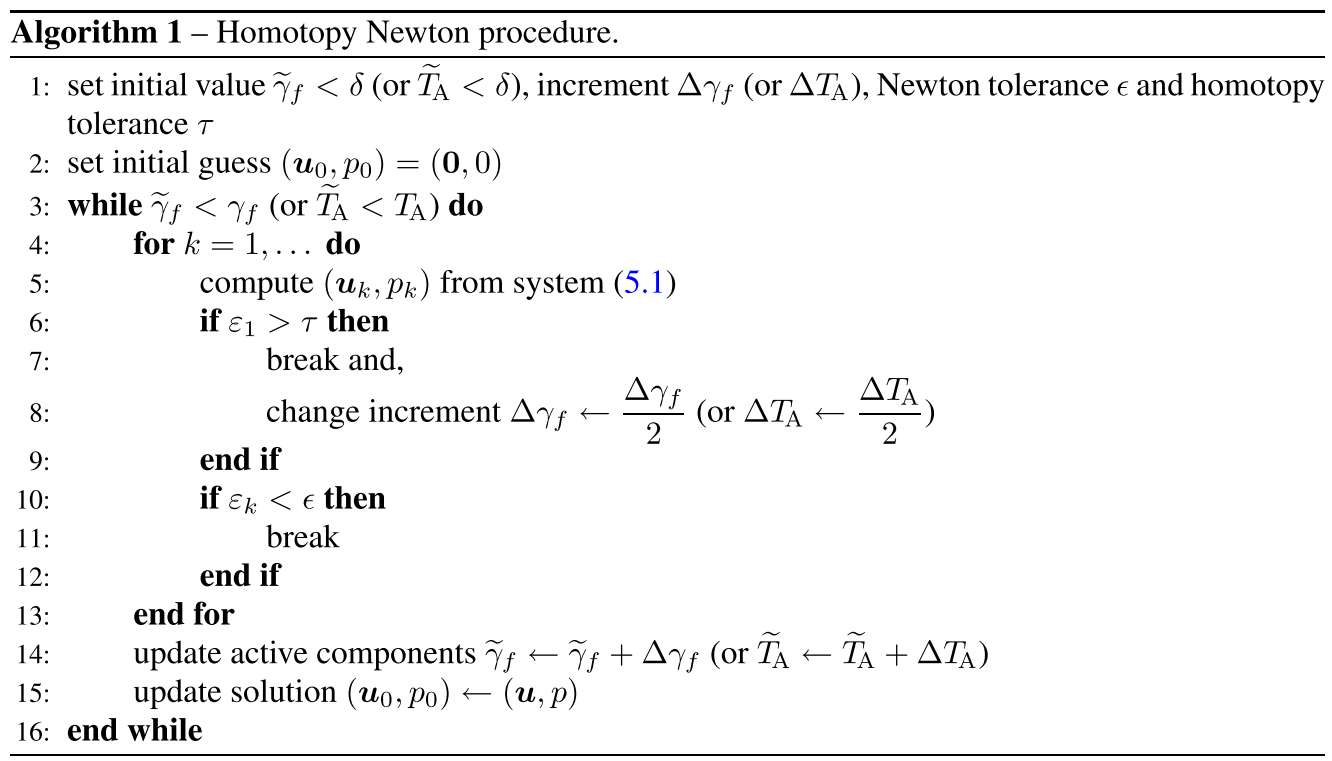

\section{NUMERICAL EXAMPLES}

We report in this section the results of numerical tests relative to some of the cases discussed in Section 3. We first start with a brief qualitative comparison between active strain and active stress formulations. Then, we proceed with some two-dimentional examples (Sections 6.2-6.4), which are not intended to assess the axial isotropy or plane stress/strain characteristic of the underlying phenomenon, but we rather include them to show the main features of the proposed formulation in these simple settings and to illustrate the performance of the numerical method under different discretization choices for both models studied. These simulations are implemented with a code using FreeFem++ [43], and numerical simulations for some three-dimentional examples in simple and anatomical settings have been carried out with a custom code in COMSOL Multiphysics (COMSOL Multiphysics 4.2 (2011), COMSOL INC. 1 New England Executive Park, Suite 350, Burlington, MA 01803, USA) [44]. All computations were ran on workstations with 64-bit processors Intel Core i7 using Nehalem microarchitectures and 4 GB of RAM.

In what follows, the action of body forces has not been considered. Moreover, Newton iterations were stopped when the total relative residual (5.2) reached the tolerance $\epsilon=10^{-10}$. For the incremental procedure, we set $\tau_{\min }=5 \cdot 10^{-3}$ and $\tau_{\max }=10^{-1}$ and imposed the increments $\Delta \gamma_{f}$ and $\Delta T_{A}$ to be $10 \%$ of the initial active component $\hat{\gamma}_{f}$ or $\hat{T}_{A}$, respectively.

\subsection{Qualitative comparison between active strain and stress approaches}

We start with three cases where the activation is given by $\exp \left\{-4(z-1 / 2)^{2}\right\}$ in the cylindrical domain $\left\{(x, y, z) \in \mathbb{R}^{3}: x^{2}+y^{2} \leq 0.25,0 \leq z \leq 1\right\}$ with fibers uniformly aligned in the $z$-direction, that is, $\mathbf{f}_{0}=(0,0,1)$. This domain represents a macroscopic piece of tissue made of myocytes and collagen sheets fully activated along the plane $z=1 / 2$. Here, we set the magnitude of the active strain to -0.3 , whereas we tune the maximum value of $T_{\mathrm{A}}$ to get the same maximum vertical displacement in both formulations. In the first two cases, we consider an isotropic behavior, neo-Hookean law (3.1) for Test A, and exponential (3.6) for Test B, whereas in Test C, we use the orthotropic law (2.2).

In Figure 4, we present the results for Test A, where the top panels show the active contribution. Clearly, the behavior of the large deformations for the two formulations is similar, whereas the pressure profiles (in the bottom panels) show different intensities. We have found that increasing the complexity of the constitutive relation also increases the differences between the displacements obtained with the two approaches. In fact, for Test B (Figure 5), such differences become more 

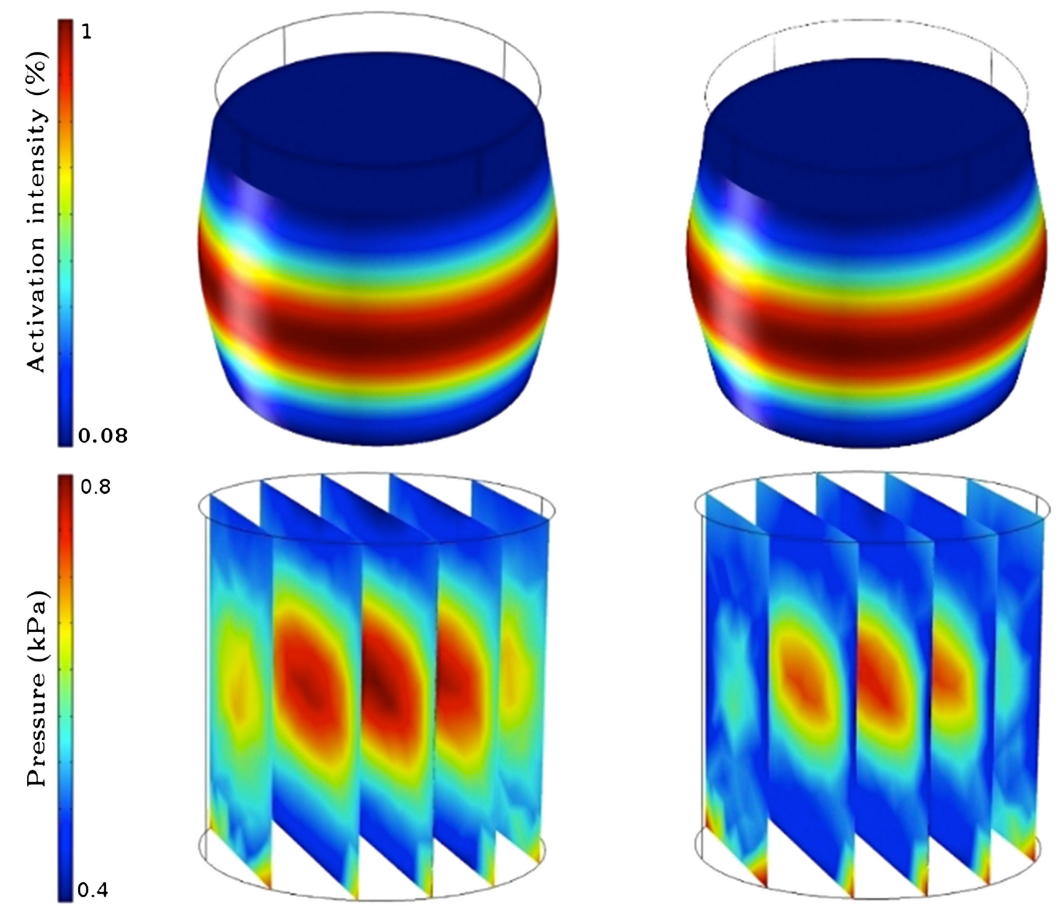

Figure 4. Test A: Intensity of the active contribution on the current configuration (top) and pressure profiles on the reference domain (bottom) obtained under active stress (left) and active strain formulations (right).

Neo-Hookean constitutive law with $\mu=0.385[\mathrm{kPa}]$.
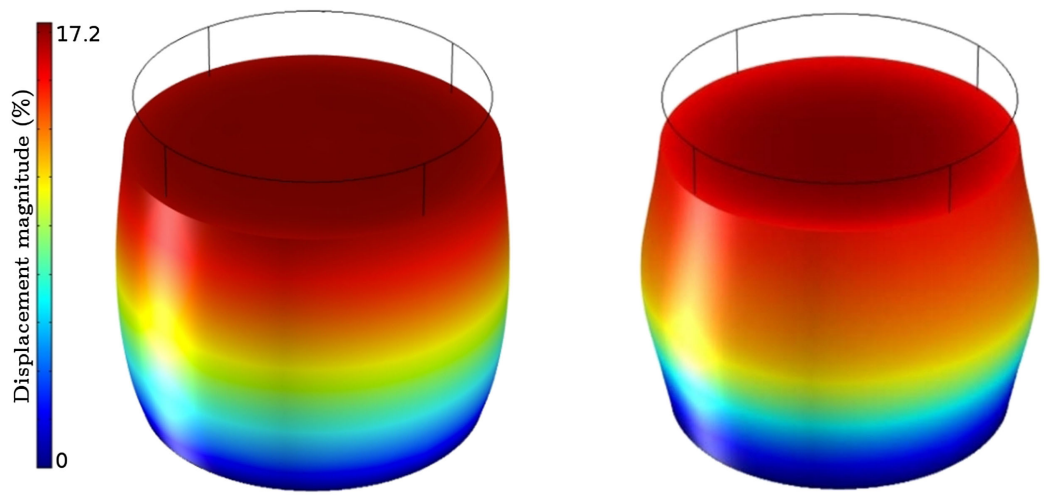

Figure 5. Test B: Displacement magnitude relative to the reference height of the cylinder on the current configuration under the exponential isotropic law with $a=0.496[\mathrm{kPa}]$ and $b=7.209$, for the active stress (left) and active strain (right) approaches. The activation is a Gaussian distribution on the $z$-axis.

apparent than those appreciated in Test A. The active contribution is a Gaussian function depending only on the $z$-coordinate with apex in the center of the cylinder. The active strain model exhibits a similar shape on the side boundaries, whereas this cannot be appreciated in the active stress model.

Results for Test $\mathrm{C}$ are shown in Figure 6, where we have imposed the sheet direction parallel to the $y$-axis. In this test, we find notable differences in displacements for the two approaches, even under the same conditions as in tests A and B. In particular, the active stress formulation is stiffer in the sheet direction, which induces a shift of most of the deformations to the sheet-normal direction $\mathbf{n}_{0}$. The anisotropy here is evident. With the active strain model, instead, we find a decreased anisotropic behavior, although present, due to the constraint $J_{\mathrm{A}}=1$. Although this condition seems to overconstrain the system, it is a natural imposition of the active isochoric motion the cells undergo. A 

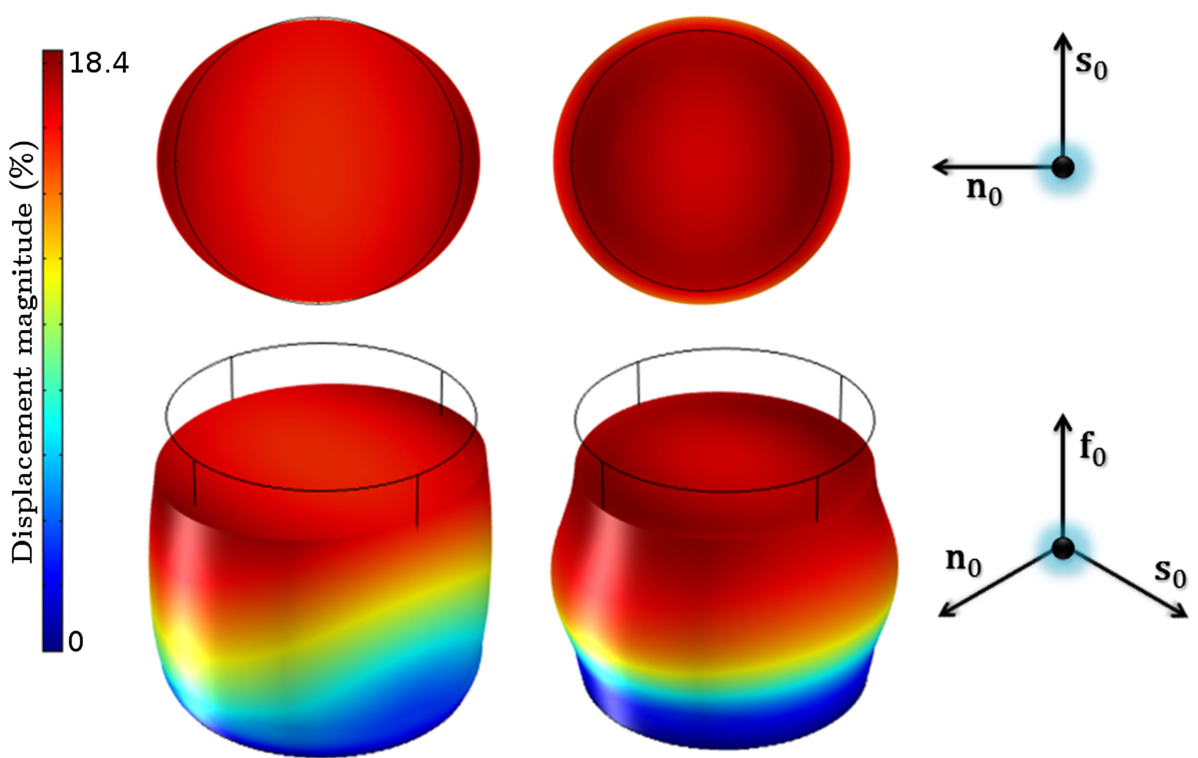

Figure 6. Test C: Displacements field for the orthotropic mechanical law where the color bar show the relative displacement with respect to the reference height of the cylinder under active stress (left) and active strain (right). The active strain formulation shows a decreased anisotropic behavior although present (maximum displacement in the sheet direction is roughly $80 \%$ than the one in the sheet-normal direction).

comparison with experimental data on the behavior of the cardiac tissue during active contraction will be important to asses the correctness of this assumption in the model. This would require to introduce in the active strain the dependence on the sarcomere length, which has been neglected in the present study. However, because the obtained results are in accordance with the discussion in Section 3.3 (we note a softening in the sheet direction under active strain, which is not present in the active stress formulation), we leave this issue for a future study.

\subsection{Example 1: Axial contraction of a neo-Hookean square}

In this example, the body is a square domain $\mathcal{B}_{0}=[0,1] \times[0,1]$ with fibers aligned to the $y$ axis, that is, $\mathbf{f}_{0}=(0,1)^{T}$. On the bottom side, we impose homogeneous Dirichlet data and stress free conditions elsewhere. The elastic modulus is $\mu=0.385$ [kPa] [30]. We set a smooth active response (see Figure 7, left) given by $T_{\mathrm{A}}=\hat{T}_{a} \exp \left[-4(y-1 / 2)^{2}\right]$, and $\gamma_{f}=\hat{\gamma}_{f} \exp \left[-4(y-1 / 2)^{2}\right]$, where $\hat{T}_{a}$ and $\hat{\gamma}_{f}$ are constants representing the maximum value of the activation. We consider $\hat{\gamma}_{f} \in\{-0.05,-0.1,-0.15,-0.2,-0.25,-0.3\}$ and $\hat{T}_{\mathrm{A}} \in\{0.13,0.26,0.39,0.52,0.65,0.78\}[\mathrm{kPa}]$, for different settings. Considering the neo-Hookean constitutive law, the deformations with active stress and active strain agree very closely, as seen in Figure 7 (top left). Table II shows the number of Newton iterations needed for convergence. These are roughly constant with respect to the level of refinement of the mesh. Taylor-Hood elements are evidently much more memory demanding (out of memory for 716,800 degrees of freedom). The values in the table were obtained with $\hat{\gamma}_{f}=-0.3$ and $\hat{T}_{\mathrm{A}}=0.78[\mathrm{kPa}]$. As expected, the rate of convergence of the incompressibility error in the $L^{2}$-norm is problem dependent. In particular, it depends on the functional form and maximum values of $\gamma_{f}$ and $T_{\mathrm{A}}$. In Figure 7, we illustrate this feature by considering various values of the active response for active stress and strain formulations. In the active strain case, we find that $\|J-1\|_{L^{2}\left(\beta_{0}\right)} \leq C h^{p_{1} \gamma_{f}+p_{2}}$, where $\left(p_{1}, p_{2}\right)=(0.6,0.66)$ for MINI elements, and $\left(p_{1}, p_{2}\right)=(1,0.59)$ for Taylor-Hood elements. Such relation is no longer linear for the active stress case. However, comparing the convergence rate with respect to the maximum vertical displacement, we found that the two formulations do not differ substantially, as expected. 

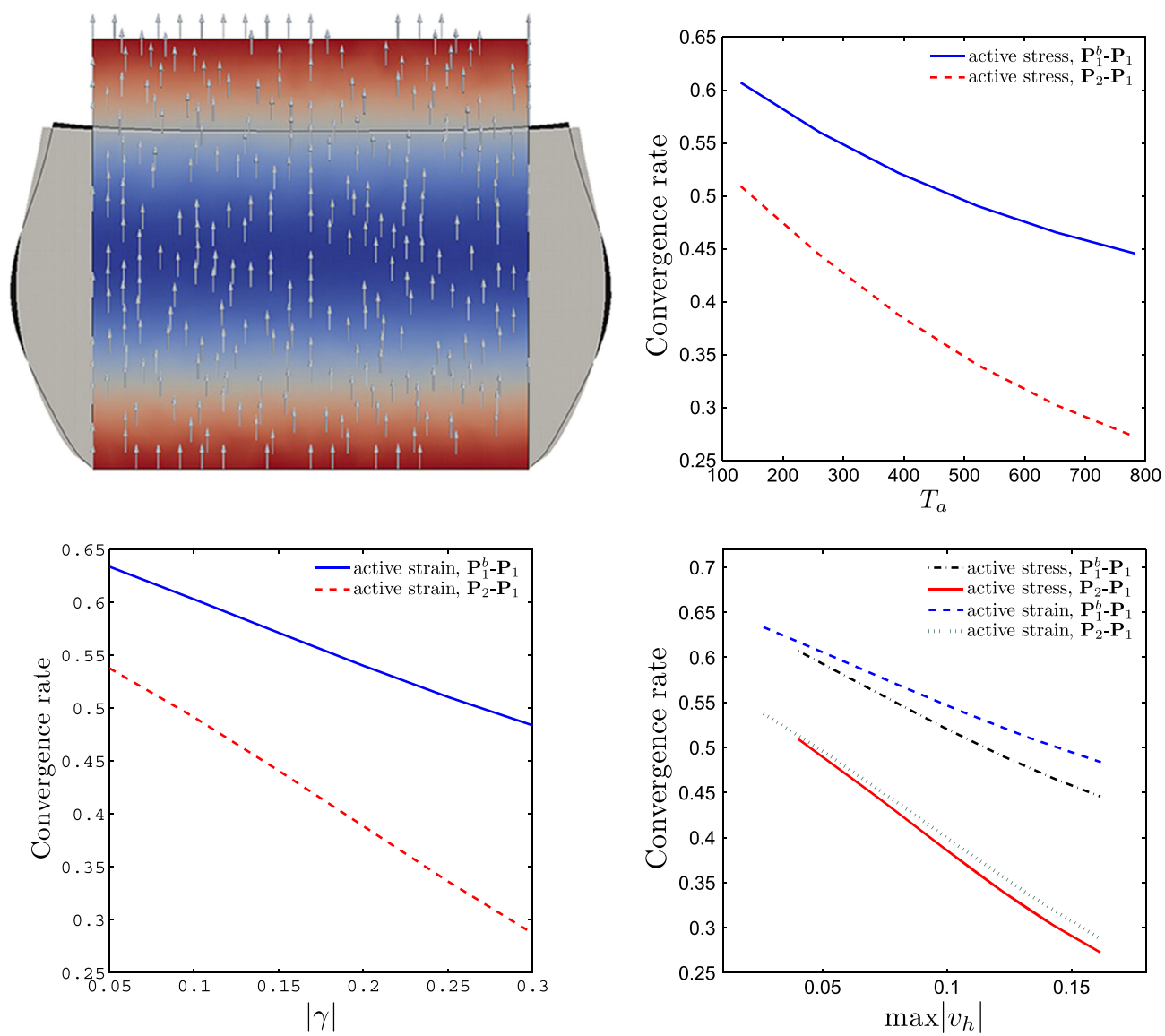

Figure 7. Example 1: Distribution of the activation and fibers on the reference domain, and comparison of the contours of the deformed domain for an active stress (gray) and strain (black) approaches (top left), and rates of convergence of the relative incompressibility error with respect to the active tension (top right), active response magnitude (bottom left), and maximum vertical displacement (bottom right), for Taylor-Hood $\left(\mathbb{P}_{2}-\mathbb{P}_{1}\right)$ and MINI $\left(\mathbb{P}_{1}^{b}-\mathbb{P}_{1}\right)$ elements.

Table II. Example 1: Newton iterations with respect to the number of elements in the mesh, for the active stress and strain formulations using Taylor-Hood $\left(\mathbb{P}_{2}-\mathbb{P}_{1}\right)$ and MINI $\left(\mathbb{P}_{1}^{b}-\mathbb{P}_{1}\right)$ elements.

\begin{tabular}{lcccc}
\hline & \multicolumn{2}{c}{$\mathbb{P}_{1}^{b}-\mathbb{P}_{1}$} & \multicolumn{2}{c}{$\mathbb{P}_{2}-\mathbb{P}_{1}$} \\
Number of elements & Active strain & Active stress & Active strain & Active stress \\
\hline 100 & 7 & 7 & 7 & 7 \\
400 & 7 & 7 & 7 & 7 \\
1600 & 7 & 7 & 7 & 7 \\
6400 & 7 & 7 & 8 & 8 \\
25,600 & 7 & 7 & 8 & 8 \\
102,400 & 8 & 8 & - & - \\
\hline
\end{tabular}

\subsection{Example 2: Neo-Hookean square with localized contraction}

In this example, we consider the same domain and boundary data as in Example 1. The fibers are now aligned with $\mathbf{f}_{0}=(\sqrt{2} / 2, \sqrt{2} / 2)^{T}$, and we impose

$$
T_{\mathrm{A}}=\left\{\begin{array}{ll}
\hat{T}_{\mathrm{A}}, & \text { if }\left(x-\frac{3}{4}\right)^{2}+(y-1)^{2} \leq \frac{1}{4}, \\
0, & \text { otherwise, }
\end{array} \quad \gamma_{f}= \begin{cases}\hat{\gamma}_{f}, & \text { if }\left(x-\frac{3}{4}\right)^{2}+(y-1)^{2} \leq \frac{1}{4}, \\
0, & \text { otherwise, }\end{cases}\right.
$$


where we set $\hat{T}_{\mathrm{A}}=0.96[\mathrm{kPa}]$ and $\hat{\gamma}_{f}=-0.3$. In Table III, we show the number of Newton iterations needed for convergence. The incremental load technique discussed in Section 5.2 was not required in Example 1, whereas here, because of the discontinuity of the activation functions (see Figure 8, left), for finer meshes, at least two incremental iterations were needed, implying an increased overall computational time. We found that Taylor-Hood elements are outperformed by MINI elements, which do not need incremental iterations. In Figure 8 (right), we display the relative incompressibility error. As expected from the preceding consideration, MINI elements exhibit a better behavior, and the convergence reaches $O\left(h^{0.5}\right)$. With this example, we also see that the convergence order is problem dependent.

\subsection{Example 3: Comparison with an exact solution in two-dimentional, neo-Hookean material}

Consider the same square domain as in Examples 1 and 2 with the fibers direction $\mathbf{f}_{0}=(0,1)^{T}$. The displacement

$$
\boldsymbol{u}=\left(\frac{\alpha}{2} y^{2}, 0\right)^{T}, \quad \mathbf{F}=\left(\begin{array}{cc}
1 & \alpha y \\
0 & 1
\end{array}\right)
$$

satisfies the incompressibility constraint.

Table III. Example 2: Newton iterations with respect to the number of elements, under active stress and active strain, using $\mathbb{P}_{2}-\mathbb{P}_{1}$ and $\mathbb{P}_{1}^{b}-\mathbb{P}_{1}$ elements. The plus signs indicate the need of incremental iterations.

\begin{tabular}{lcccc}
\hline \multirow{2}{*}{ Number of elements } & \multicolumn{2}{c}{$\mathbb{P}_{1}^{b}-\mathbb{P}_{1}$} & \multicolumn{2}{c}{$\mathbb{P}_{2}-\mathbb{P}_{1}$} \\
& Active strain & Active stress & Active strain & Active stress \\
\hline 141 & 6 & 6 & 7 & 7 \\
517 & 7 & 7 & 7 & 10 \\
1978 & 7 & 7 & 8 & 8 \\
6561 & 7 & 7 & $8+4$ & 10 \\
25,921 & 7 & 8 & $8+4$ & $10+4+4+4$ \\
103,041 & 8 & 8 & - & - \\
\hline
\end{tabular}
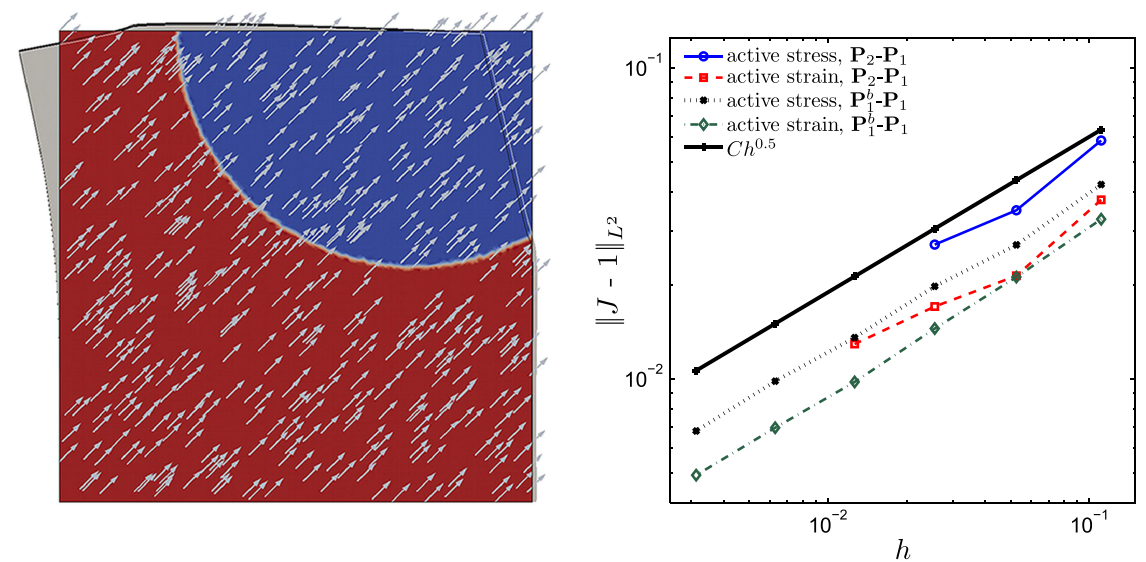

Figure 8. Example 2: Distribution of the activation function and fibers on the reference domain, and comparison of the contours of the deformed domain for an active stress (gray) and active strain (black) approaches (left). Relative incompressibility error with respect to the number of elements for active stress and strain formulations with $\mathbb{P}_{2}-\mathbb{P}_{1}$ and $\mathbb{P}_{1}^{b}-\mathbb{P}_{1}$ elements (right). 
The Piola-Kirchhoff tensors in both formulations read

$$
\begin{aligned}
& \mathbf{P}_{\text {stress }}=\mu \mathbf{F}+T_{\mathrm{A}} \mathbf{F} \mathbf{f}_{0} \otimes \mathbf{f}_{0}-p J \mathbf{F}^{-T}, \\
& \mathbf{P}_{\text {strain }}=\mu\left(1+\gamma_{f}\right) \mathbf{F}-\mu\left(\gamma_{f}+\gamma_{f} \frac{\gamma_{f}+2}{\left(1+\gamma_{f}\right)^{2}}\right) \mathbf{F} \mathbf{f}_{0} \otimes \mathbf{f}_{0}-p J \mathbf{F}^{-T},
\end{aligned}
$$

and they can be merged in the general stress

$$
\mathbf{P}=\mu\left(1+\gamma_{f}\right) \mathbf{F}-\left[\mu\left(\gamma_{f}+\gamma_{f} \frac{\gamma_{f}+2}{\left(1+\gamma_{f}\right)^{2}}\right)+T_{\mathrm{A}}\right] \mathbf{F f}_{0} \otimes \mathbf{f}_{0}-p J \mathbf{F}^{-T},
$$

where

$$
\left(T_{\mathrm{A}}, \gamma_{f}\right)= \begin{cases}\left(\hat{T}_{\mathrm{A}}, 0\right), & \text { for active stress } \\ \left(0, \hat{\gamma}_{f}\right), & \text { for active strain. }\end{cases}
$$

We set $\hat{T}_{\mathrm{A}}=0.96[\mathrm{kPa}]$ and $\hat{\gamma}_{f}=-0.3$, and inserting (6.1) in the balance equations, we find

$$
p(x, y)=\frac{\alpha^{2}}{2}\left[\frac{\mu}{\left(1+\gamma_{f}\right)^{2}}+T_{\mathrm{A}}\right] y^{2}+\alpha\left[\frac{\mu}{\left(1+\gamma_{f}\right)^{2}}+T_{\mathrm{A}}\right] x+K,
$$

for some constant $K$. For the simulation, we use $\alpha=2, K=0, \boldsymbol{u}=\mathbf{0}$ on $\Gamma_{D}=[0,1] \times\{0\}$ and traction data $\mathbf{P n}=\boldsymbol{t}$ on the remaining boundaries, where $\boldsymbol{t}=\mathbf{P}_{\text {exact }} \mathbf{n}$ is written using the exact stress tensor computed from (6.1). The value of $\alpha$ determines the magnitude of the deformations, and a too large value may lead to non-convergence. In such cases, the incremental technique of Sections 5.2 and 5 can be applied to $\alpha$ instead of the activation function.

In Figure 9, we report the relative errors for all fields. For this particular case, a fast convergence of $O\left(h^{5.5}\right)$ for $\boldsymbol{u}, O\left(h^{4.8}\right)$ for $p$ and $O\left(h^{5.2}\right)$ for $J$ is observed.

\subsection{Example 4: Comparison with an exact solution in three-dimentional, Holzapfel-Ogden material}

Let us consider now the constitutive law (2.2) applied to the cylindrical domain

$$
\mathcal{B}_{0}=\left\{(x, y, z) \in \mathbb{R}^{3}:\left|x^{2}+y^{2}\right| \leq \frac{1}{4}, 0 \leq z \leq 1\right\},
$$

with the fibers and sheets aligned in the directions $\mathbf{f}_{0}=(0,0,1)^{T}$ and $\mathbf{s}_{0}=(0,1,0)^{T}$, respectively. To check the convergence of the method, we use the exact solution $\boldsymbol{u}=(\alpha z, \beta z, \delta)$, which satisfies the incompressibility constraint, and we set $\alpha=1 / 4, \beta=1 / 8, \delta=0$. The invariants are explicitly given by

$$
I_{1}=3+\alpha^{2}+\beta^{2}, I_{4, f}=1+\alpha^{2}+\beta^{2}, I_{4, s}=1, I_{8, f s}=\beta,
$$
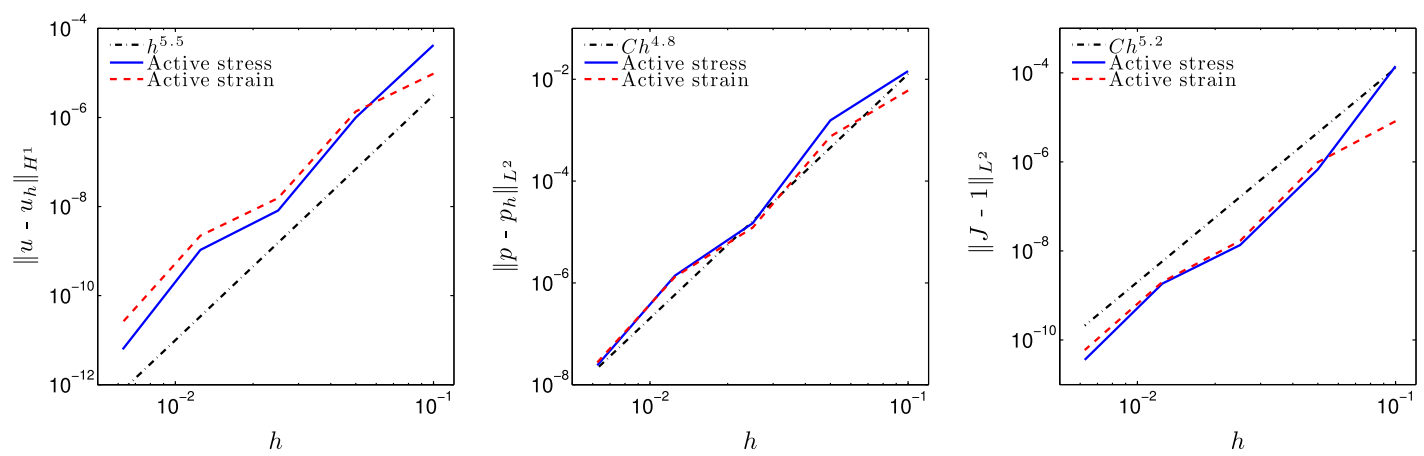

Figure 9. Example 3: Relative errors for velocity, pressure, and incompressibility, using Taylor-Hood $\left(\mathbb{P}_{2}-\mathbb{P}_{1}\right)$ elements. 
and the transformed invariants read

$$
\begin{aligned}
& I_{1}^{\mathrm{E}}=\left(3+\alpha^{2}+\beta^{2}\right)\left(1+\gamma_{f}\right)+\left(1+\alpha^{2}+\beta^{2}\right)\left(\gamma_{f}+\gamma_{f} \frac{\gamma_{f}+2}{\left(1+\gamma_{f}\right)^{2}}\right), \\
& I_{4, f}^{\mathrm{E}}=\frac{\left(1+\alpha^{2}+\beta^{2}\right)}{\left(1+\gamma_{f}\right)^{2}}, \quad I_{4, s}^{\mathrm{E}}=\left(1+\gamma_{f}\right), \quad I_{8, f s}^{\mathrm{E}}=\frac{\beta}{\sqrt{1+\gamma_{f}}} .
\end{aligned}
$$

We can write the stress tensor of both models as

$$
\begin{aligned}
\mathbf{P}_{\text {stress }} & =\left(\begin{array}{ccc}
2 \psi_{1}-p & \alpha \psi_{8, f s} & \alpha\left(2 \psi_{1}+2 \psi_{4 f}+T_{\mathrm{A}}\right) \\
0 & 2 \psi_{1}+\beta \psi_{8, f s}-p & \beta\left(2 \psi_{1}+2 \psi_{4 f}+T_{\mathrm{A}}\right)+\psi_{8, f s} \\
\alpha p & \psi_{8, f s}+\beta p & 2 \psi_{1}+2 \psi_{4 f}+T_{\mathrm{A}}-p
\end{array}\right), \\
\mathbf{P}_{\text {strain }} & =\left(\begin{array}{ccc}
2 \psi_{1}^{\mathrm{E}}-p & \alpha \psi_{8, f s}^{\mathrm{E}} & \alpha\left(2 \psi_{1}^{\mathrm{E}}+2 \psi_{4 f}^{\mathrm{E}}\right) \\
0 & 2 \psi_{1}^{\mathrm{E}}+2 \psi_{4 s}^{\mathrm{E}}+\beta \psi_{8, f s}^{\mathrm{E}}-p & \beta\left(2 \psi_{1}^{\mathrm{E}}+2 \psi_{4 f}^{\mathrm{E}}\right)+\psi_{8, f s}^{\mathrm{E}} \\
\alpha p & \psi_{8, f s}^{\mathrm{E}}+\beta p & 2 \psi_{1}^{\mathrm{E}}+2 \psi_{4 f}^{\mathrm{E}}-p
\end{array}\right) .
\end{aligned}
$$

In both cases, from $\operatorname{div} \mathbf{P}=\mathbf{0}$, we find that $p(x, y, z)=K$, for some constant $K$. We impose the Dirichlet boundary conditions $\boldsymbol{u}=(0,0, \delta)^{T}$ on the bottom face, and we set Neumann conditions according to the exact solution on the remaining boundaries, that is, $\mathbf{P n}=\boldsymbol{t}$, with $\boldsymbol{t}=\mathbf{P}_{\text {exact }} \mathbf{n}$ being computed directly from the exact solution. In addition, we set $K=2.689[\mathrm{kPa}]$, $T_{\mathrm{A}}=a=0.496[\mathrm{kPa}]$, and $\gamma_{f}=-0.1$. Lower values of $\gamma_{f}$ may lead to an ill-conditioned problem because the functions $\psi_{i}^{\mathrm{E}}, i=1,4 f, 4 s, 8 f s$ depend exponentially on the active strain, and this has no minor effects on the boundary conditions. In Figure 10 (left), we show the qualitative behavior of the solution, and in Figure 10 (right), the relative incompressibility errors exhibiting a slow convergence rate of $O\left(h^{0.65}\right)$. In Table IV, we show the Newton iterations needed for convergence. For the two finest meshes, the active stress formulation requires about twice Newton iterations, because at least one more incremental iteration is needed. In practice, the active stress formulation will require even more incremental steps if larger deformations are considered.
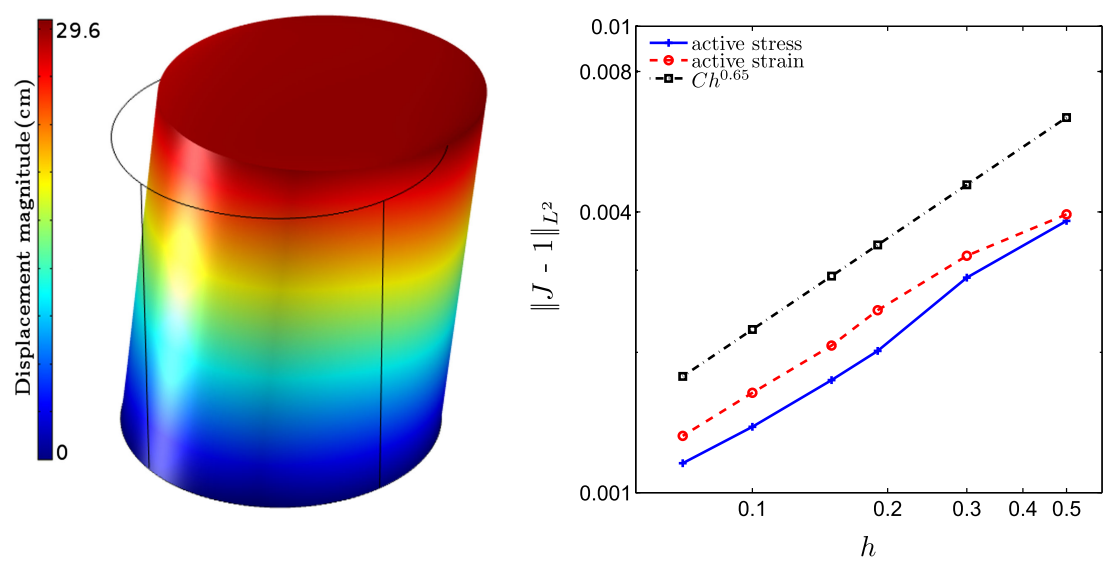

Figure 10. Example 4: Numerical solution obtained with the active strain formulation (left), and convergence history for the relative incompressibility errors (right).

Table IV. Example 4: Newton iterations for convergence with the active stress and strain formulations using Taylor-Hood elements. The plus signs indicate the need of incremental iterations.

\begin{tabular}{lcccccc}
\hline Number of elements & 214 & 433 & 1381 & 3928 & 12,765 & 251,112 \\
\hline Active strain & 8 & 8 & 9 & 8 & 8 & 8 \\
Active stress & 9 & 9 & 10 & 12 & $9+6$ & $10+6$ \\
\hline
\end{tabular}




\subsection{Example 5: Simulations on an idealized biventricular domain}

We have constructed an idealized biventricular domain using end-diastolic geometrical data reported in $[45,46]$. The inner surface of the left ventricle (lv) and right ventricle (rv) are described by the ellipsoids

$$
\frac{x^{2}}{a_{\mathrm{lv}}^{2}}+\frac{y^{2}}{b_{\mathrm{lv}}^{2}}+\frac{z^{2}}{c_{\mathrm{lv}}^{2}}=1, \quad \frac{x^{2}}{a_{\mathrm{rv}}^{2}}+\frac{y^{2}}{b_{\mathrm{rv}}^{2}}+\frac{z^{2}}{c_{\mathrm{rv}}^{2}}=1,
$$

where $a_{\mathrm{lv}}=b_{\mathrm{lv}}=2.4[\mathrm{~cm}], c_{\mathrm{lv}}=4.5[\mathrm{~cm}]$, and $a_{\mathrm{rv}}=3.4[\mathrm{~cm}], b_{\mathrm{rv}}=5.8[\mathrm{~cm}], c_{\mathrm{rv}}=4.7[\mathrm{~cm}]$. The ellipsoids are truncated to have an apex-to-base distance of 6 [cm]. On the left ventricle, we impose a wall thickness of $1.5[\mathrm{~cm}]$ at the base and $0.5[\mathrm{~cm}]$ at the apex. The right ventricle wall thickness was set to $0.3[\mathrm{~cm}]$ near the apex, whereas, at the base, it was set to $0.5[\mathrm{~cm}]$ near the left ventricle and $0.4[\mathrm{~cm}]$ away from it.

We $\mathbf{s}_{0}=\left(x / \sqrt{x^{2}+y^{2}}, y / \sqrt{x^{2}+y^{2}}, 0\right)$ as an approximation for the sheets direction. The fiber direction $\mathbf{f}_{0}$ is defined orthogonal to $\mathbf{s}_{0}$ in the planes $z=$ constant; and it is rotated by an angle of $-45^{\circ}$ with respect to the sheet axis to get an approximate direction on the outer surface. We define the distance from the outer wall $\Delta r$, and we use it to rotate the fiber direction through the thickness of the wall by an angle of $90^{\circ}$. The radius of the ellipsoids are set by substituting the parametric equations $x=r \cos \theta \cos \phi, y=r \cos \theta \sin \phi, z=r \sin \theta$ in (6.2) and solving for $r$. Denoting the outer radii of the left and right ventricles by $r_{\mathrm{lv}}$ and $r_{\mathrm{rv}}$, respectively, we define the distances from the outer walls as $\Delta r_{\mathrm{lv}}=r_{\mathrm{lv}}-R$ and $\Delta r_{\mathrm{rv}}=r_{\mathrm{rv}}-R$, with $R=|\mathbf{x}|$. All this gives the following relations for the angle of rotation with respect to the sheet axis inside the muscle walls $\theta_{\mathrm{lv}}=m_{\mathrm{lv}} \Delta r_{\mathrm{lv}}$, and $\theta_{\mathrm{rv}}=m_{\mathrm{rv}} \Delta r_{\mathrm{rv}}$, with $m_{\mathrm{lv}}=112.2$ and $m_{\mathrm{rv}}=392.7$.

The final configuration of our idealized biventricular domain is portrayed in Figure 11. The mesh consists of 1983 tetrahedral elements. We set zero Dirichlet boundary conditions in a small area on the base wall separating the two ventricles and free stress boundary conditions $(\mathbf{P n}=\mathbf{0})$ on the remaining boundaries. We define

$$
T_{\mathrm{A}}=\hat{T}_{\mathrm{A}} \exp \left(-500\left[x^{2}+(y+0.03)^{2}+(z)^{2}\right]\right), \gamma_{f}=\hat{\gamma}_{f} \exp \left(-500\left[x^{2}+(y+0.03)^{2}+(z)^{2}\right]\right) .
$$

The homotopy procedure was required in these simulations. The initial values of the activation are set to $T_{\mathrm{A}}^{i}=0.1[\mathrm{KPa}]$ and $\gamma_{f}^{i}=-0.1$, and the final values of the activation were $T_{\mathrm{A}}^{\mathrm{max}}=50[\mathrm{KPa}]$ and $\gamma_{f}^{\max }=-0.3$. The active stress formulation required roughly 25 incremental iterations compared with only five iterations needed for active strain, indicating that in this framework, the active strain may lead to a decreased computational cost.

In Figure 12 (left), the pressure profiles on selected sections of the reference configuration are shown. Both formulations present similar results in accordance with [23]. In the remaining plots of Figure 12, we observe the magnitude of displacements and corresponding deformed domain from different angles (the arrows show the fiber vectorial field) for the active strain (top) and stress (bottom). The structure of the domain and the complex configuration of the fibers determine an
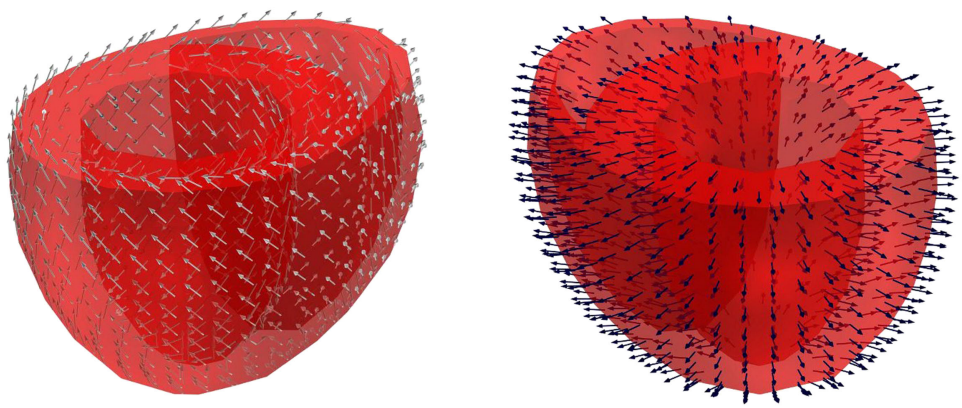

Figure 11. Example 5: Imposed fibers (left) and sheets (right) directions in the undeformed idealized biventricular domain. 

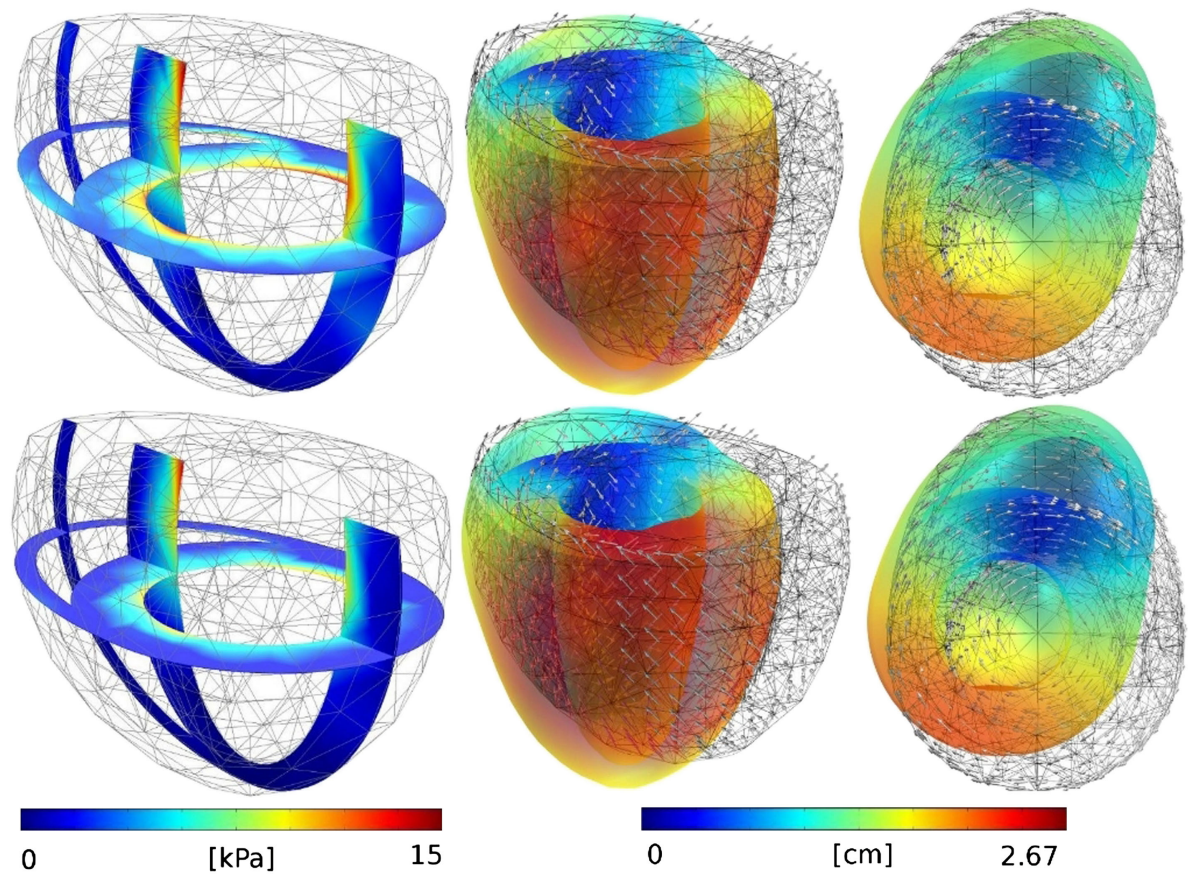

Figure 12. Example 5: Pressure profiles on selected sections of the reference configuration (left) and two different views of the displacement magnitude on the deformed domain and fibers distribution on the undeformed mesh (middle and right), for active strain (top) and active stress (bottom) models.
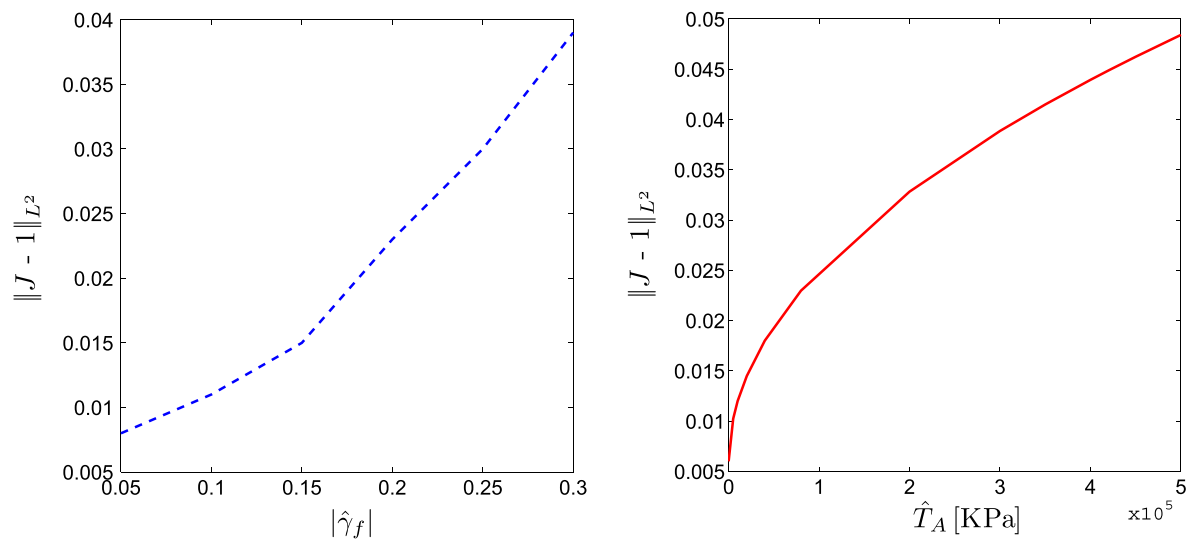

Figure 13. Example 5: Relative errors on $J$ with respect to the active strain magnitude $\hat{\gamma}_{f}$ (left) and active tension $\hat{T}_{A}$ (right) for a test on the ideal biventricular domain shown in Figure 11.

apex-based twist together with the contraction. We remark that even if the imposed boundary conditions are not able to reproduce physiological regimes, here we can still appreciate a torsion of the cardiac muscle. Moreover, with the active strain approach, we obtain a smaller left ventricle diameter contraction. It has decreased from $4.8[\mathrm{~cm}]$ to $3.2[\mathrm{~cm}]$, against the $3.75[\mathrm{~cm}]$ obtained with the active stress formulation. Anyhow, both results are in accordance with end-systolic data in $[45,46]$. The incompressible nature of the simulation with the imposed boundary conditions requires that for a decrease in the left ventricle diameter, we obtain an apex-to-base elongation. Conversely, an apex-to-base contraction would lead to an increase of the ventricles diameter. Such solution can be retrieved by imposing a vertical fiber direction in the mid-wall.

A sensibility study is reported in Figure 13, where we plot the relative incompressibility error with respect to the magnitude of the maximum value of the activation functions. More evident differences are observed between the active stress and strain approaches: Increasing the active strain 
magnitude also increases almost 'linearly' the magnitude of the deformation, while on the other hand, increasing the active tension 'saturates' the deformations, in the sense given in the picture. As in consequence, for a larger deformation to be obtained, a very high value for $\hat{T}_{\mathrm{A}}$ is required, which of course translates in a substantial increase in the number of incremental load iterations.

\subsection{Example 6: Simulations on a canine heart geometry}

As a final example, we present simulation results performed on a canine biventricular geometry reconstructed from segmented MRI images [47] (Figure 14). The apex-to-base distance in the left ventricle is roughly $5.5[\mathrm{~cm}]$, and its diameter varies between 2.1 and $2.4[\mathrm{~cm}]$. The wall thickness in the left ventricle is approximately $0.5-0.8[\mathrm{~cm}]$, whereas for the right ventricle, it is around 0.4 [cm]. The mesh consists of 30,309 tetrahedral elements. The average direction of the fibers goes from an angle of $45^{\circ}$ in the epicardium to $-45^{\circ}$ in the endocardium; and as in Example 5, the sheet directions are oriented approximately normal to the endocardium and epicardium. We use the orthotropic constitutive law with the parameters as in Table I and constant activation $\gamma_{f}=-0.2$. To mimic the response of active strain, we impose an active tension of $T_{\mathrm{A}}=17$ [kPa]. We fix a small region in the inter-ventricular base while the rest of the boundary remains free. Taylor-Hood finite elements are used, and the algorithm converged after 18 Newton iterations for active strain and 21 for active stress, both using incremental steps.

In Figure 15, we depict pressure profiles and several views of the displacement magnitude on the deformed domain, along with the reference undeformed mesh for a computation with the active stress and active strain models. A clear contraction of the apex is obtained. The results of both models agree with experimental observations [12] in terms of torsion of the left ventricle (basal torsional rotation of around $12^{\circ}$ and apical around $-12^{\circ}$, from a basal view). To further assess the physiological relevance of our computations, we compare principal and shear strains in the fibersheet-normal coordinates to experimental results performed on canine populations: experiment 1 [3] and experiment 2 [48] (we consider their results obtained at the end-systolic phase, because it represents a quasi-steady state where the mechanical load of myocytes is near maximal [49]). We see from Figure 16 a fair agreement between computed and observed data, specially taking into consideration previous comparisons of experiments with numerical results obtained with orthotropic active stress models [5]. Here, for instance, we observe that the active strain model is closer to experimental data for the normal strains in the fiber direction $\mathbf{f}$ in the endocardial and epicardial region, while the active stress formulation gives a better approximation in the mid-wall region. In the directions $\mathbf{s}$ and $\mathbf{n}$, the results obtained under active strain are always closer to the observed data (of both experiments) than those from active stress (see Figure 16, top). In addition, the shear strains are higher under active strain than under active stress, as mentioned in Section 3.3, whereas it is unclear which model recovers better the results from experiment 2 (strains in the mixed directions, Figure 16 bottom).
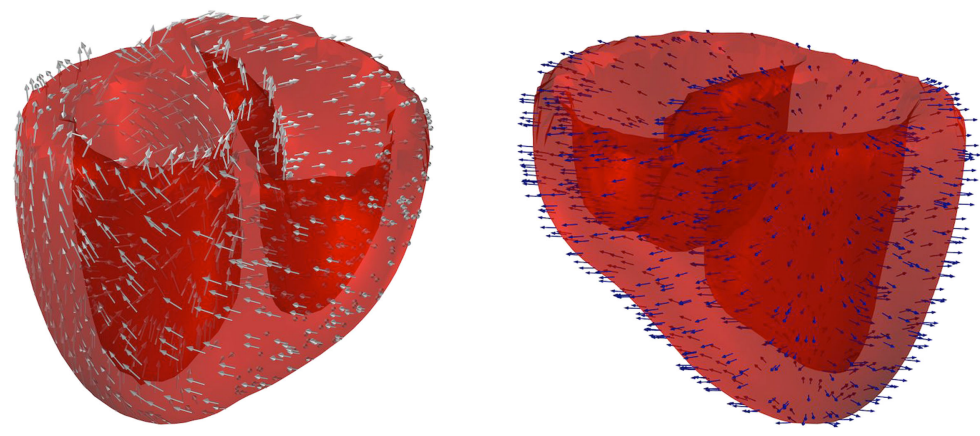

Figure 14. Example 6: Fibers (left) and sheet (right) distribution in the reference configuration. 


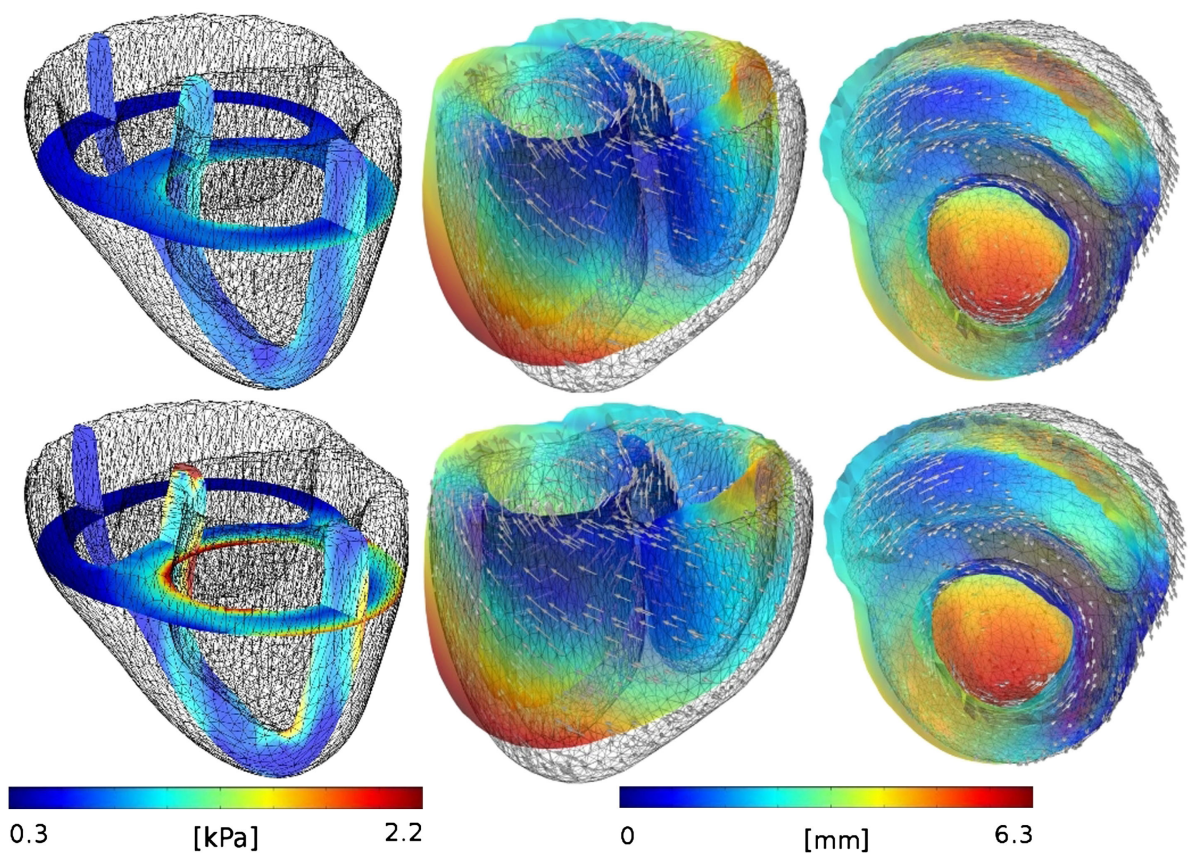

Figure 15. Example 6: Pressure profiles on selected sections of the reference configuration (left) and two different views of the displacement magnitude on the current configuration and fibers in the undeformed mesh for an active stress (top) and active strain model (bottom).
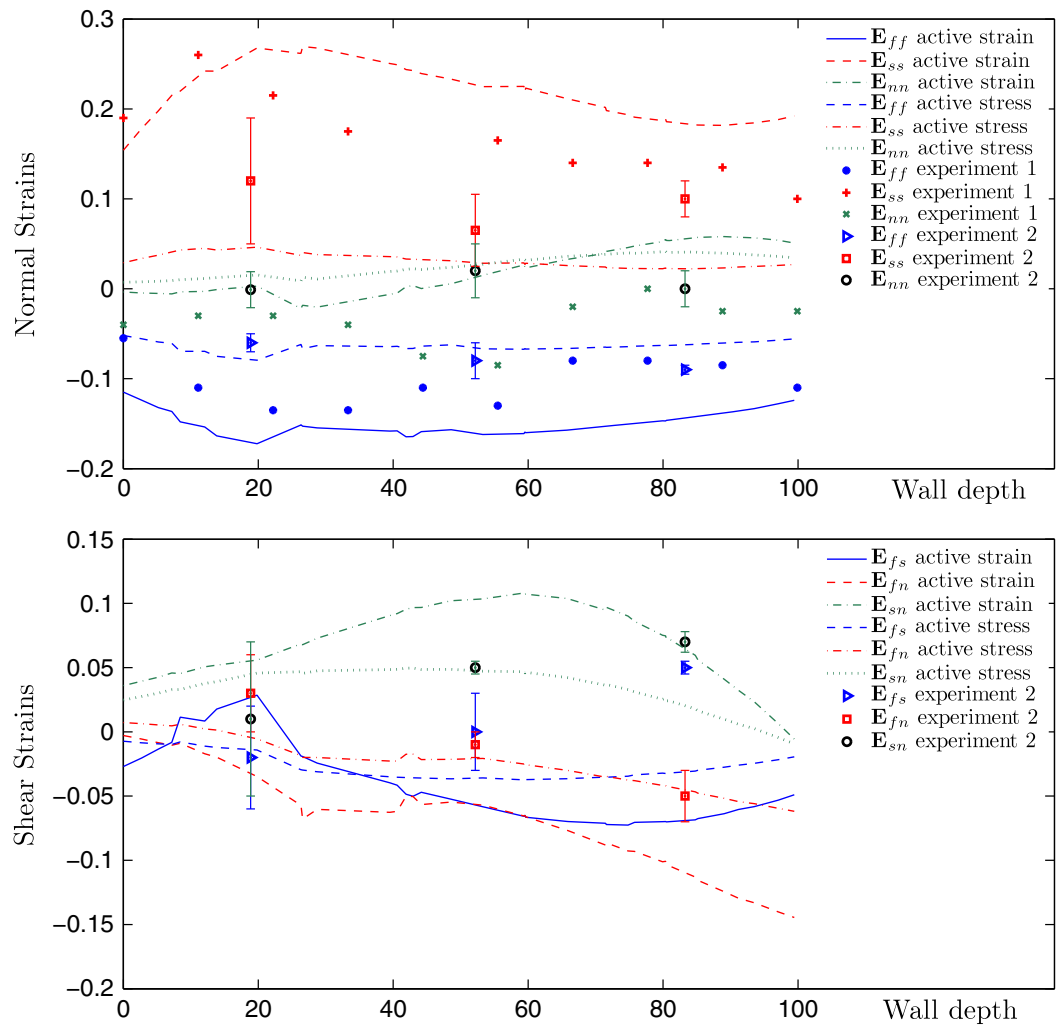

Figure 16. Example 6: Comparison of computed transmural distributions of strains in fiber-sheet coordinates at a basal site and experimental data from end-systolic canine in vivo tests in experiments 1 and 2 (from [3] and [48], respectively). Wall depth of 0\% stands for endocardium and 100\% for epicardium. 


\section{CONCLUSION}

In this paper, we have presented some advances on the modeling of cardiac mechanics in the context of the recently proposed active strain formulation introducing it in the framework of orthotropic material laws. We have shown that for isotropic constitutive laws, our model produces similar results as the active stress approach in terms of displacements and pressure. On the other hand, for orthotropic materials, the two formulations lead to different responses. The passive myocardium was assumed to obey the constitutive law given by [20] for volume-preserving deformations. The macroscopic orthotropic passive mechanics of the tissue was coupled with a transversely isotropic active component at the cell scale. Moreover, we have performed a thorough qualitative comparison with the active stress approach from the modeling and numerical viewpoints, considering diverse configurations and different constitutive laws in two and three spatial dimensions. Our numerical method is based on a Newton linearization of the original system combined with an incremental activation technique, and the spatial discretization is carried out using piecewise linear finite elements for the approximation of the pressure field, whereas for the displacements, we use piecewise quadratic elements or alternatively piecewise linear finite elements enriched with bubble functions.

Even though we compared our results with torsion and deformations data obtained from medical images, and experimental tests, the question of whether the active strain formulation gives more satisfactory results from the standpoint of physiology remains to be addressed in further detail. As pointed out in [16], the active stress model possesses more flexibility, in the sense that the parameters and the tensorial dependence of the active part of the stress tensor can be tuned to represent experimental data. In the active strain formulation, we do not have such flexibility in the definition of the active component. This may be regarded as a limitation in the sense that once the form of the active deformation $\mathbf{F}_{\mathrm{A}}$ is imposed, it is not possible to adjust stresses to fit data coming from experimental observations.

The fact that activated tissue possesses different mechanical properties in all directions suggests that a more general active stress model should be used (as discussed in Section 2.2 and in $[5,15]$ ). The assumption that the active response may be described as hyperelastic, although not true in general [25], leads to a simple definition of the active forces: the new active strain energy for the active stress formulation can be found by fitting data from active uniaxial and biaxial tests. With the active strain approach, instead, the passive constitutive law is somehow naturally modified (the active component $\gamma_{f}$ modifies directly the material response in all directions), leading to a new energy function that is able to capture active responses. Moreover, this new energy exhibits similar stability properties as the original one, whereas an active stress model may lead to instabilities due to the loss of strong ellipticity.

Regarding numerical aspects, we have found that an active strain formulation does not require any added computational cost. Moreover, even if the studied models present similar convergence properties with respect to the mesh size and need a similar number of Newton iterations to converge for large deformations, the active stress model will require many more incremental active load iterations. In the end, taking as benchmark the active stress formulation as the most widely used model for electro-mechanical simulations of cardiac tissue, the active strain model proposed here has proven to give satisfactory qualitative results and to be a competitive alternative. The framework developed in this paper allows for a natural coupling with the equations governing the propagation of electrical potential through the medium in the direction of $[12,14]$. A forthcoming contribution will address the construction of domain decomposition methods that can be used to couple models with variable mechanical properties (thickness, conductivities, elastic moduli) across the muscle wall $[2,50]$.

\section{ACKNOWLEDGEMENTS}

The authors thank Davide Ambrosi and Simone Pezzuto for stimulating discussions on the subject of this paper. In addition, Simone Rossi acknowledges support by the IST-EPFL Joint Doctoral Initiative, and Alfio Quarteroni and Ricardo Ruiz-Baier are supported by the European Research Council through the advanced grant "Mathcard, Mathematical Modelling and Simulation of the Cardiovascular System", ERC-2008-AdG 227058. 


\section{REFERENCES}

1. Humphrey JD. Cardiovascular Solid Mechanics: Cells, Tissues, and Organs. Springer-Verlag: New York, 2002.

2. Arts T, Costa KD, Covell JW, McCulloch AD. Relating myocardial laminar architecture to shear strain and muscle fiber orientation. American Journal of Physiology. Heart and Circulatory Physiology 2001; 280:H2222-H2229.

3. Waldman LK, Fung YC, Convell JW. Transmural myocardial deformation in the canine left ventricle: normal in vivo three-dimensional finite strains. Circulatory Research 1985; 57:152-163.

4. Nash MP, Hunter PJ. Computational mechanics of the heart. Journal of Elasticity 2000; 61:113-141.

5. Usyk T, Mazhari R, McCulloch AD. Effect of laminar orthotropic myofiber architecture on regional stress and strain in the canine left ventricle. Journal of Elasticity 2000; 61:143-164.

6. Kelly D, Mackenzie L, Hunter P, Smaill B, Saint DA. Gene expression of stretch-activated channels and mechanoelectric feedback in the heart. Clinical and Experimental Pharmacology and Physiology 2006; 33: 642-648.

7. Kohl P, Hunter P, Noble D. Stretch-induced changes in the heart rate and rhythm: clinical observations, experiments and mathematical models. Progresses in Biophysics and Molecular Biology 1999; 71:91-138.

8. Murtada S, Kroon M, Holzapfel GA. A calcium-driven mechanochemical model for prediction of force generation in smooth muscle. Biomechanics and Modeling in Mechanobiology 2010; 9:749-762.

9. Göktepe S, Kuhl E. Electromechanics of the heart: a unified approach to the strongly coupled excitation-contraction problem. Computational Mechanics 2010; 45:227-243.

10. Pathmanathan P, Chapman SJ, Gavaghan D, Whiteley JP. Cardiac electromechanics: the effect of contraction model on the mathematical problem and accuracy of the numerical scheme. Quarterly Journal of Mechanics and Applied Mathematics 2010; 63:375-399.

11. Sainte-Marie J, Chapelle D, Cimrman R, Sorine M. Modeling and estimation of the cardiac electromechanical activity. Computers and Structures 2006; 84:1743-1759.

12. Evangelista A, Nardinocchi P, Puddu PE, Teresi L, Torromeo C, Varano V. Torsion of the human left ventricle: experimental analysis and computational modelling. Progresses in Biophysics and Molecular Biology 2011; 107:112-121.

13. Nardinocchi P, Teresi L. On the active response of soft living tissues. Journal of Elasticity 2007; 88:27-39.

14. Nobile F, Quarteroni A, Ruiz-Baier R. An active strain electromechanical model for cardiac tissue. International Journal for Numerical Methods in Biomedical Engineering 2012; 28:52-71. DOI: 10.1002/cnm.1468.

15. Lin DHS, Yin FCP. A multiaxial constitutive law for mammalian left ventricular myocardium in steady-state barium contracture or tetanus. Journal of Biomechanical Engineering 1998; 120:504-518.

16. Ambrosi D, Pezzuto S. Active stress vs. active strain in mechanobiology: constitutive issues. Journal of Elasticity DOI: 10.1007/s10659-011-9351-4. to appear.

17. Brezzi F, Fortin M. Mixed and hybrid finite element methods. Springer-Verlag: New York, 1991.

18. Auricchio F, Beira̧o da Veiga L, Lovadina C, Reali A. A stability study of some mixed finite elements for large deformation elasticity problems. Computer Methods in Applied Mechanics and Engineering 2005; 194:1075-1092.

19. Pantuso D, Bathe KJ. On the stability of mixed finite elements in large strain analysis of incompressible solids. Finite Elements in Analysis and Design 1997; 104:28-83.

20. Holzapfel GA, Ogden RW. Constitutive modelling of passive myocardium: a structurally based framework for material characterization. Philosophical Transactions of the Royal Society of London. Series A 2009; 367:3445-3475.

21. Yin FCP, Chan CCH, Judd RM. Compressibility of perfused passive myocardium. American Journal of Physiology. Heart and Circulatory Physiology 1996; 271:H1864-H1870.

22. Chapelle F, Gerbeau JF, Sainte-Marie J, Vignon-Clementel IE. A poroelastic model valid in large strains with applications to perfusion in cardiac modeling. Computational Mechanics 2010; 46:91-101.

23. Göktepe S, Acharya SNS, Wong J, Kuhl E. Computational modeling of passive myocardium. International Journal for Numerical Methods in Biomedical Engineering 2011; 27:1-12.

24. Le Tallec P. Existence and approximation results for nonlinear mixed problems: application to incompressible finite elasticity. Numerische Mathematik 1982; 38:365-382.

25. Gibbs CL, Mommaerts WFHM, Ricchiuti NV. Energetics of cardiac contractions. The Journal of Physiology 1967; 191:25-46.

26. Papademetris X, VSkrinjar O, Duncan JS. Recovering displacements and deformations from 3D medical images using biomechanical models. In Handbook of Numerical Analysis, Ayache N (ed.). Elsevier, 2004; 551-590.

27. Niederer SA, Hunter PJ, Smith NP. A quantitative analysis of cardiac myocyte relaxation: a simulation study. Biophysical Journal 2005; 90:1697-1722.

28. Rice JJ, Wang F, Bers DM, de Tombe PP. Approximate model of cooperative activation and crossbridge cycling in cardiac muscle using ordinary differential equations. Biophysical Journal 2005; 95:2368-2390.

29. Lee EH, Liu DT. Finite strain elastic-plastic theory with application to plane-wave analysis. Journal of Applied Physics 1967; 38:17-27.

30. Menzel A, Steinmann P. On configurational forces in multiplicative elastoplasticity. International Journal of Solids and Structures 2007; 44:4442-4471.

31. Simo JC. A framework for finite strain elastoplasticity based on maximum plastic dissipation and the multiplicative decomposition: part 1. Continuum formulation. Computer Methods in Applied Mechanics and Engineering 1988; 66:199-219.

32. Taber LA, Yang M, Podszus WW. Mechanics of ventricular torsion. Journal of Biomechanics 1996; 29:745-752. 
33. Stålhand J, Klarbring A, Holzapfel GA. A mechanochemical 3D continuum model for smooth muscle contraction under finite strains. Journal of Theoretical Biology 2011; 268:120-130.

34. Rausch MK, Dam A, Göktepe S, Abilez OJ, Kuhl E. Computational modeling of growth: systemic and pulmonary hypertension in the heart. Biomechanics and Modeling in Mechanobiology 2011; 10:799-811. DOI: 10.1007/s10237-010-0275-x.

35. Jewell BR. A reexamination of the influence of muscle length on myocardial performance. Circulation Research 1977; 40:221-230.

36. Simmons RM, Jewell BR. Mechanics and models of muscular contraction. Recent Advances in Physiology 1974; 9:87-147.

37. Iribe G, Helmes M, Kohl P. Force-length relations in isolated intact cardiomyocytes subjected to dynamic changes in mechanical load. American Journal of Physiology. Heart and Circulatory Physiology 2007; 292:H1487-H1497.

38. Kamgoué A, Ohayon J, Usson Y, Riou L, Tracqui P. Quantification of cardiomyocyte contraction based on image correlation analysis. Cytometry A 2009; 75:298-308.

39. Walton J, Wilber J. Sufficient conditions for strong ellipticity for a class of anisotropic materials. International Journal of Non-Linear Mechanics 2003; 38:441-455.

40. Davis TA, Duff IS. An unsymmetric-pattern multifrontal method for sparse LU factorization. SIAM Journal on Matrix Analysis and Applications 1997; 18:140-158.

41. Amestoy PR, Duff IS, L'Excellent JY. Multifrontal parallel distributed symmetric and unsymmetric solvers. Computer Methods in Applied Mechanics and Engineering 2000; 184:501-520.

42. Crisfield MA. Non-linear Finite Element Analysis of Solids and Structures. John Wiley \& Sons: Chichester, 1991.

43. Hecht F. FreeFem++, (3rd edn). Laboratoire Jacques-Louis Lions: Université Pierre et Marie Curie, Paris, 2008.

44. COMSOL Multiphysics 4.2 (2011), COMSOL INC. (Available from: http://www.comsol.com/) [Accessed on March 2011].

45. Basavarajaiah S, Wilson M, Naghavi R, Whyte G, Turner M, Sharma S. Physiological upper limits of left ventricular dimensions in highly trained junior tennis players. British Journal of Sports Medicine 2007; 41:784-788.

46. Talley NJ, O’Connor S. Examination Medicine, 6th ed. Churchill Livingstone: Sydney, 2010.

47. Peyrat JM, Sermesant M, Pennec X, Delingette H, Xu C, McVeigh ER, Ayache N. A computational framework for the statistical analysis of cardiac diffusion tensors: application to a small database of canine hearts. IEEE Transactions on Medical Imaging 2007; 26:1500-1514.

48. Takayama Y, Costa KD, Covell JW. Contribution of laminar myofiber architecture to load-dependent changes in mechanics of left ventricular myocardium. American Journal of Physiology. Heart and Circulatory Physiology 2002; 282:H1510-H1520.

49. Solomon SD, Aikawa Y, Martini MS, Rosario L, Makker G, Gerson D, Greaves S, Lee RT. Assessment of regional left ventricular wall stress after myocardial infarction by echocardiography-based structural analysis. Journal of the American Society of Echocardiography 1998; 11:938-947.

50. Bajaj C, Goswami S. Multi-component heart reconstruction from volumetric imaging. Proceedings of the ACM Solid and Physical Modeling Symposium, Stony Brook, NY, 2008; 193-202. 